\title{
Evidence for conformational change-induced hydrolysis of $\beta$-tubulin-GTP
}

Mohammadjavad Paydar ${ }^{1}$ and Benjamin H. Kwok ${ }^{1 \dagger}$

${ }^{1}$ Institute for Research in Immunology and Cancer (IRIC), Département de médecine, Université de Montréal, P.O. Box 6128, Station Centre-Ville, Montréal, QC H3C 3J7, Canada.

${ }^{\dagger}$ Corresponding author:

B. H. Kwok: benjamin.kwok@umontreal.ca

\section{Keywords:}

Kinesin, Kif2A, MCAK, motor protein, microtubules, tubulin, GTP hydrolysis

\author{
Abbreviations \\ NM: Neck+Motor \\ MD: Motor domain \\ MT: Microtubule
}

Running title: Tubulin GTP hydrolysis 


\begin{abstract}
Microtubules, protein polymers of $\alpha / \beta$-tubulin dimers, form the structural framework for many essential cellular processes including cell shape formation, intracellular transport, and segregation of chromosomes during cell division. It is known that tubulin-GTP hydrolysis is closely associated with microtubule polymerization dynamics. However, the precise roles of GTP hydrolysis in tubulin polymerization and microtubule depolymerization, and how it is initiated are still not clearly defined. We report here that tubulin-GTP hydrolysis can be triggered by conformational change induced by the depolymerizing kinesin- 13 proteins or by the stabilizing chemical agent paclitaxel. We provide biochemical evidence that conformational change precedes tubulin-GTP hydrolysis, confirming this process is mechanically driven and structurally directional. Furthermore, we quantitatively measure the average size of the presumptive stabilizing "GTP cap" at growing microtubule ends. Together, our findings provide the molecular basis for tubulin-GTP hydrolysis and its role in microtubule polymerization and depolymerization.
\end{abstract}




\section{INTRODUCTION}

Microtubules (MTs), protein polymers of $\alpha / \beta$-tubulin dimers, are a major component of the cell cytoskeleton. MTs provide the framework for giving a cell its shape, for intracellular transport and for segregating the chromosomes during cell division. The formation of MT polymers and the regulation of their dynamics in space and time are crucial for their functions during different phases of the cell cycle. The fundamental questions of how these polymers are formed and their dynamics controlled at the molecular level are still not fully understood.

\section{MT polymerization and tubulin-Guanosine triphosphate (GTP) hydrolysis. Microtubule}

polymerization occurs when $\alpha / \beta$-tubulin subunits join together to form a polymer of hollow tubule [1]. Polymerization is initiated by a process called nucleation - the assembly of two or more tubulin dimers. This can happen spontaneously or build on pre-existing templates such as the $\gamma$-tubulin ring complex [2-4]. Once the nucleus is formed, more subunits can be added to form filaments and are self-organized into a hollow tubular structure. It has been well established that MT polymerization is a stochastic process, with polymers cycling between periods of growing and shrinking. This phenomenon is known as dynamic instability [5].

It was known early on that MT polymerization requires GTP [6-8] and that $\alpha / \beta$-tubulin dimers, subunits of MTs are GTPases. In their nucleotide-binding pockets, $\alpha$-tubulin contains a non-interchangeable GTP constitutively while $\beta$-tubulin has a hydrolysable and exchangeable GTP [9-11]. GTPs in $\beta$-tubulin subunits are hydrolyzed during MT polymerization and within the MT lattice in two steps: from GTP to guanosine diphosphate(GDP)- $\mathrm{P}_{\mathrm{i}}$ and then to GDP [12, 13]. GTP hydrolysis is presumed to contribute to MT polymerization dynamics and regulating the catastrophe switch [14], but the underlying molecular mechanism is still poorly understood.

Early EM study showed that polymerizing ends are often blunt and straight while the depolymerizing ends are curved or tapered [15]. This led to the pleasingly simple hypothesis that GTP-tubulins are straight, and the GDP tubulins are curved. The straight conformation of GTPtubulin facilitates the incorporation of dimers into polymer ends forming the stabilizing GTP cap and the formation of lateral bond with adjacent protofilaments. As polymerization proceeds, GTP gets hydrolyzed and the GDP-tubulins in the MT middle are constrained to the straight conformation and are inherently unstable $[16,17]$. Upon removal of the stabilizing GTP cap, the GDP-tubulin dimers are exposed and the constrains are released, leading to the outward curved 
1 of the depolymerizing filament. This model is appealing because it explains most of biochemical

2 and conformational events associated with microtubule polymerization and depolymerization.

3 However, accumulating structural studies have found that both GTP- and GDP-tubulin dimers

4 are curved [18-20], and that curved or tapered filaments are also observed in polymerizing

5 microtubule ends [21-23]. In light of these findings, it is conceivable that curved GTP-tubulins

6 are added to the polymerizing ends. As polymerizing ends grow and filaments straighten through

7 interaction with adjacent filaments, GTP hydrolysis ensues. Recent high resolution cryo-electron

8 microscopy (cryo-EM) studies on MT polymers stabilized with taxol or hydrolysis-resistant

9 GMPCPP have suggested that GTP-bound tubulin stabilized the polymers by strengthening the

10 lateral and longitudinal interactions between neighboring tubulin subunits, and that hydrolysis

11 leads to the destabilization GDP-tubulin in the lattice [16, 17, 24]. Upon depolymerization, the

12 previously constrained dimers return to their naturally curved state, and the energy stored in the constrained tubulin dimer is released to do mechanical work. While this revised model is logical and sound, it is still puzzling as to what triggers GTP hydrolysis and why since GTP hydrolysis per se is not needed for the addition of GTP-tubulin subunit at MT ends.

GTP cap and MT stability. Being enriched in GTP-bound tubulin dimers, the growing end of microtubules is often referred to as "GTP cap"; this structure is thought to stabilize MTs [12, 25]. It has also been proposed that some GTP-bound tubulin dimers may exist in MT middle (GTP islands), due to incomplete GTP hydrolysis during polymerization [12, 25]. Evidence for this proposal has been obtained indirectly using a conformation-specific antibody [26]. The islands of GTP-bound tubulin have been speculated to be responsible for MT rescue, switching MTs from shortening to growing $[26,27]$. The length/size of the GTP cap has been a mystery and controversial for decades. Earlier studies suggested the GTP cap size could be as small as a single GTP-tubulin layer [1, 28, 29]. This is, however, inconsistent with recent findings by indirect probing with MT end-binding (EB) proteins, which have been shown to bind preferentially to GTP-bound tubulin [30-33]. There have been efforts using this feature to determine the GTP cap size in vivo, and some have reported it to contain hundreds of tubulin dimers ( $~ 750$ dimers spread over $\sim 55$ rows) [34, 35]. It has been proposed that the length of EB comet tail is closely linked to the growth rate and stability of MTs [31, 36, 37], strengthening the 
1 idea that the GTP cap stabilizes the MT polymers. Curiously, in a more recent study, the length

2 of outwardly curved MT ends was measured to be about $\sim 40-80 \mathrm{~nm}$ in vitro and also in cells

3 from different species, using EM tomography [22]. It is not known, however, whether the

4 curvature is linked to the nucleotide-bound state of tubulin dimers associated with the

5 protofilament ends.

MT dynamics in cells. In cells, MT dynamics are largely regulated by MT associated proteins (MAPs). Tubulin post-translational modifications have also been shown to regulate different MT features, including their dynamics, by altering their binding affinity to MAPs [38, 39]. Some of these proteins promote MT polymerization/stabilization, like Stu2/XMAP215/Dis1 family polymerases, which contain multiple TOG domains that bind $\alpha \beta$-tubulin dimers [40]. Another member of polymerizing MAPs is TPX2, which is known as an MT catastrophe suppresser [41]. On the other hand, MT depolymerizing MAPs are also important in controlling MT dynamics. Members of Kinesin-8 family are MT destabilizing motors that move towards the plus-end of MTs. The depolymerization mechanism of Kinesin-8 motor proteins, however, varies between different species [42-44]. In contrast, kinesin-13 family members (KIF2A, KIF2B and KIF2C/MCAK) are major catastrophe factors that actively remove subunits from MT ends [45, 46]. These proteins hydrolyze adenosine triphosphate (ATP) when they encounter tubulin dimers, free or in the polymers. However, their depolymerizing function only happens at the ends of MTs, where they use the energy derived from ATP hydrolysis to dissociate tubulin dimers [47, 48]. Our structural study shows each kinesin-13 monomer can bind to two tubulin dimers in tandem and bend both the intra- and inter-dimer curvature [49]. We postulate that this extreme bending of tubulin dimers by kinesin-13 ultimately leads to their dissociations from protofilament ends.

We report here that kinesin-13 proteins induce a conformational change in tubulin dimers, which in turn triggers the hydrolysis of the exchangeable GTP on $\beta$-tubulins. We provide

27 experimental evidence to show that it is the conformational change of tubulin that leads to GTP hydrolysis and not the other way around. We further demonstrate that this structural change of tubulin dimer has to be directional, from a more curved to a straighter conformation and not vice versa, to trigger GTP hydrolysis. Furthermore, the same mechanism also occurs during tubulin 
1 polymerization. Finally, we provide a molecular account for the occurrence of GTP-tubulin associated MT ends, often termed the "GTP cap", and quantitatively measure its average length. In sum, our work presented here reveals the inner workings of tubulin biochemistry: the molecular relationship between structural change and nucleotide hydrolysis.

\section{RESULTS}

Tubulin-GTP hydrolysis can be triggered by binding and unbinding of kinesin-13 proteins. The presence of GDP in $\beta$-tubulin, in our previous structural study of the KIF2A-tubulin ternary complex [49], prompted us to determine if the hydrolysis of tubulin GTP occurred spontaneously during the sample preparation and crystallization processes, or if it was induced by forming the complex with KIF2A-NM (neck+motor; amino acids 153-553). To address this directly, we first used a fluorescence-based assay to detect the presence of GDP over time when tubulin dimers either were incubated alone or with KIF2A-NM in the presence of AMP-PNP and DARPin (Designed Ankyrin Repeat Protein, a polypeptide that binds to $\beta$-tubulin and prevents MT polymerization). Our data showed that tubulin-GTP turns over very slowly on its own (with a rate of $0.0003 \mathrm{~S}^{-1}$ ), but the hydrolysis rate increased significantly when incubated with KIF2A$\mathrm{NM}$ in the presence of AMP-PNP and DARPin (with an initial rate of $0.16 \mathrm{~S}^{-1}$,Fig. 1A). Note that tubulin dimers incubated with AMP-PNP-bound KIF2A alone or with DARPin alone did not promote GTP hydrolysis. From our previous study [49], we knew that DARPin could compete with the neck region of KIF2A-NM for binding to $\beta$-tubulin and thereby release KIF2a-NM from the complex, facilitating GTP turnover. Therefore, excess DARPin should disrupt the complex formation and suppress GTP hydrolysis. Indeed, DARPin titration in the same experimental setting yielded the result that is consistent with this prediction (Fig. 1B).

Our results showed that GTP hydrolysis required the associated tubulin dimers to be released from AMP-PNP-bound KIF2A-NM (i.e. bound only with DARPin, which disrupts the 1:2 KIF2A:tubulin dimer complex, as previously shown [49]). This suggests that the bending of tubulin dimers by KIF2A-NM per se does not trigger GTP hydrolysis; instead it is the return of free tubulin dimers to their native conformation that promotes the activity. If this assertion is correct, ATP hydrolysis by KIF2A, stimulated by tubulin dimers, should also trigger GTP hydrolysis. As DARPin was used only to facilitate the crystallization of KIF2A-NM tubulin 
complex, it should not be needed for KIF2A or kinesin-13 proteins to interact with tubulin dimers at MT ends, there they induce MT depolymerization. To ensure that what we observed was not due to an artifact of using DARPin to force the release of KIF2A-NM from tubulin dimer, and to determine if kinesin-13-induced tubulin-GTP hydrolysis also occurs in the presence of ATP, we performed the same experiment in the presence of ATP. As anticipated, this was indeed the case (Fig. 1C). Our result showed that GTP turnover also occurred in the presence of ATP, as robustly as or even more robust than that with AMP-PNP and DARPin. Although the GDP detection reagent that we used has a lower sensitivity to adenosine diphosphate (ADP) (see calibration curve in Fig. S1), the signal is measurable and therefore should be subtracted from the total signal (Fig. 1C, solid and dotted red lines represent before and after background subtraction, using the determined ATP turnover rate). The results obtained with ATP were comparable to those with AMP-PNP and DARPin. To obtain further evidence on this point, we performed the same experiment using radio-labeled $\gamma^{32} \mathrm{P}-\mathrm{GTP}$ as a tracer to unambiguously distinguish GTP hydrolysis from ATP hydrolysis. This experiment showed a time-dependent increase of GTP hydrolysis (Fig. 1D-E), consistent with the result from the GDP detection assay (Fig. 1C). As a control, we monitored the basal tubulin-GTP turnover over longer periods of time, using radio-labeled $\gamma^{32} \mathrm{P}-\mathrm{GTP}$, which showed slow basal GTP hydrolysis of $0.0002\left(\mathrm{~s}^{-1}\right)$ (Fig. S2), also consistent with the rate measured by GDP detection assay. Monitoring tubulin-GTP hydrolysis in the presence of another kinesin-13 construct, MCAK-NM, yielded results almost identical to those with KIF2A-NM (Fig. S3).

\section{Tubulin-GTP hydrolysis occurs during MT depolymerization via catalytic depolymerization by} kinesin-13s, but not by non-catalytic means. Kinesin-13 proteins, including KIF2A and MCAK, are known to depolymerize MTs $[48,50]$. We wondered if the observed induction of GTPtubulin hydrolysis occurs during MT depolymerization when kinesin-13s encounter tubulin at MT ends. To address this, we performed kinesin-13 (KIF2A-NM and MCAK-NM)-induced depolymerization assay using MT polymers that retained their GTP-bound like state. MTs can be made with slowly hydrolyzable GTP analogs, guanosine 5-3-O-(thio)triphosphate (GTP $\gamma \mathrm{S})$ or guanylyl-(alpha, beta)-methylene-diphosphonate (GMPCPP). Hydrolysis of GTP $\gamma \mathrm{S}$ produces GDP, just as GTP hydrolysis does. It has been shown that kinesin-13 proteins can depolymerize 
MT depolymerization, we first generated ${ }^{35}$ S-labeled GTP $\gamma$ S MTs. To reduce background 2 radioactivity from unpolymerized tubulin and excess ${ }^{35} \mathrm{~S}-\mathrm{GTP} \gamma \mathrm{S}$, we separated the ${ }^{35} \mathrm{~S}-$ labeled GTP $\gamma \mathrm{S}$ MT polymers from the rest of the reaction mixture by ultracentrifugation through a glycerol cushion. We resuspended the radio-labeled MTs in stabilizing PIPES-based buffer and used it in a kinesin-13 mediated depolymerization reaction. We resolved the GTP $\gamma \mathrm{S}$ hydrolysis via thin layer chromatography (TLC) and monitored the level of MT depolymerization by sedimentation assay. From this experiment, we could observe concomitant GTP $\gamma \mathrm{S}$ hydrolysis and MT depolymerization with both KIF2A-NM and MCAK-NM (Fig. 2A-C \& Fig. S4). This result indicates that the depolymerization of these MTs by kinesin-13s can trigger GTP $\gamma \mathrm{S}$ hydrolysis. Analogous experiment with GMPCPP MTs in kinesin-13-mediated depolymerization using a phosphate detection assay yielded similar result indicating that hydrolysis of GMPCPP also occurred upon MT depolymerization (Fig. S5). This observation is intriguing because kinesin-13-induced MT depolymerization is even strong enough to force the hydrolysis of GTP $\gamma$ S or GMPCPP, which normally does not occur during the polymerization process. This raises the possibility that this unusual hydrolysis occurs either when MT undergoes depolymerization, or only when conformational change is specifically induced by kinesin-13 proteins. To distinguish these two possibilities, we sought to depolymerize MTs by alternative means. Exposure to $\mathrm{Ca}^{2+}$ or cold temperature is known to cause MT depolymerization, including those MTs formed with GTP $\gamma \mathrm{S}$ or GMPCPP $[23,52,53]$. For this, we set up depolymerization reactions using ${ }^{35} \mathrm{~S}$-labeled GTP $\gamma \mathrm{S}$ MTs. Interestingly, both $\mathrm{Ca}^{2+}$ and cold treatments did not trigger GTP $\gamma \mathrm{S}$ hydrolysis (marked by the lack of ${ }^{35} \mathrm{~S}$-labeled $\mathrm{P}_{\mathrm{i}}$ release, Fig. $2 \mathrm{D}$ top \& Fig.-2E) despite their high efficiency in depolymerizing MTs (indicated by tubulin dimer release in supernatant Fig. 2D bottom \& Fig.2F). Together, these experiments indicate that the depolymerization of MTs induced by kinesin-13 proteins, but not by calcium or cold treatment, causes a conformational change of tubulin dimers severe enough to trigger hydrolysis of GTP $\gamma \mathrm{S}$ or GMPCPP, and by inference, GTP as well.

Interdependent relationship between kinesin-13 ATPase rate and tubulin-GTP turnovers. complex with two tubulin dimers in tandem and changes their curvatures, but we do not know whether this interaction triggers hydrolysis of both GTP molecules or just one. Our crystal 
structure suggests one of the two dimers is more curved than the other when they bind to KIF2A-

2 NM with AMP-PNP. However, we do not have information as to whether the degrees of curvature are the same in the presence of ATP and whether the change(s) in curvature of either or both dimer(s) is (are) sufficient to trigger tubulin-GTP hydrolysis upon release. To address

5 this, we first set up nucleotide hydrolysis experiments with KIF2A-NM or MCAK-NM and free tubulin dimers with radio-labeled $\alpha^{32} \mathrm{P}-\mathrm{ATP}$ and $\gamma^{32} \mathrm{P}-\mathrm{GTP}$ tracers, and resolved samples by TLC (Fig. 3A). From these experiments, we could quantitatively measure how much ATP and GTP hydrolyzed in the same reaction based on the amount of released radio-labeled $\alpha^{32} \mathrm{P}-\mathrm{ADP}$ and ${ }^{32} \mathrm{P}_{\mathrm{i}}$, respectively (Fig. 3B). We consistently observed that for each ATP hydrolyzed, there was approximately twice as much GTP hydrolyzed. In parallel experiments, we used the same set up and added both radio-labeled tracers in the same reaction mix, and we obtained the same result (Fig. S6). These data indicate that upon each encounter, for each ATP molecule hydrolyzed by KIF2A-NM, there is a corresponding hydrolysis of two GTP molecules from the two associated tubulin dimers. This also suggests that both tubulin dimers, within the ternary complex, undergo conformational changes that are severe enough to trigger tubulin-GTP hydrolysis upon their releases from the associated kinesin-13 protein.

It has also been shown that MT polymers stimulate kinesin-13 ATPase activity more than free tubulin dimers [48]. We hypothesized that straighter tubulin conformations stimulate kinesin-13 ATPase activity more than the curved ones. To test this, we set up kinesin-13 ATPase assays in the presence of tubulin dimers with different guanine nucleotides (GTP, GTP $\gamma$ S, GDP or Apo-state). Consistent with this hypothesis, we observed that GTP- or GTP $\gamma \mathrm{S}$-tubulin dimers stimulated kinesin-13 ATPase turnovers significantly higher than those with GDP or the Apostate using three independent detection assays (radioactivity, malachite green-based phosphate detection assay and ADP-Glo ${ }^{\mathrm{TM}}$ assay). These data are summarized in Table 1 and Fig. S7A-B. This interpretation is based on the published structural data indicating that GTP-tubulin dimers (PDB code: 4DRX) are slightly straighter than GDP-tubulin (PDB code: 1SAO) [54-56]. We postulated that these differences in ATPase rate might be due to the corresponding differences in the binding affinity of tubulin dimers in different nucleotide states to kinesin-13 proteins. To test this, we set up a binding assay using tubulin dimers in different nucleotide states with either Histagged KIF2A-NM or MCAK-NM, which could be captured by Ni-charged magnetic beads. As expected, tubulin dimers associated with GTP- or GTP $\gamma \mathrm{S}$ - showed significantly higher binding 
affinity to both KIF2A-NM or MCAK-NM, than those with GDP- or no nucleotide (Apo) (Fig. 2 S7C-E).

Based on the ATPase rates of KIF2A-NM and the corresponding tubulin-GTPase rates

4 summarized in Table 1, we could also observe the 1:2 ratio that we had determined earlier in Fig.

5 3. Together, these results demonstrated the interdependent relationship between kinesin-13 ATPase activity and tubulin-GTP turnovers as well as the associated tubulin conformational

7 states.

Conformational change-induced tubulin-GTP hydrolysis during MT polymerization. Our

finding, that conformational change in tubulin dimers can trigger GTP hydrolysis in our kinesin13 induced experiments, prompted us to consider the possibility that conformation-induced tubulin-GTP turnover also occurs during MT polymerization. It is known that both GTP-tubulin and GDP-tubulin dimers are more curved compared to the straight conformation of tubulin subunits within the MT lattice except for those at polymer ends. We postulated that the reason GTP $\gamma$ S and GMP-CPP tubulins promote MT polymerization is because they are already in a straighter conformation and that the change in conformation is not big enough to trigger hydrolysis of these slowly hydrolyzable GTP analogs. Likewise, paclitaxel promotes tubulin polymerization in the presence of GTP by straightening the associated free tubulin dimers. If this assessment is true, we should be able to observe paclitaxel-induced tubulin-GTP hydrolysis prior to polymerization. To test this possibility directly, we set up experimental conditions where tubulin-GTP and paclitaxel were both present at low concentrations to disfavor spontaneous polymerization, at $5 \mu \mathrm{M}$ and 1-4 $\mu \mathrm{M}$ respectively. To further ensure that polymerization did not occur readily, we ran the experiment at $4{ }^{\circ} \mathrm{C}$, using the GDP detection assay. As anticipated, we observed almost instantaneous conversion of GTP to GDP in the presence of paclitaxel but not at its absence (Fig. 4A). We also noted that the plateau of GDP accumulation was reached quickly and never exceeded the concentrations of paclitaxel used. We verified that there was no

27 detectable level of MT polymerization under these conditions by ultracentrifugation-based sedimentation assay (Fig. S8). Together, these data suggest that paclitaxel binding triggers a conformational change of free tubulin dimers from their native curvature to a straighter state in the absence of polymer formation. We postulated that if this assertion is correct, paclitaxelstabilized tubulin-GDP might also be able to form polymer. This was observed when we 
attempted tubulin-GDP polymerization in the presence of paclitaxel (Fig. 4B). The polymers that

formed from tubulin-GDP are essentially the same as those formed form tubulin-GTP as assessed by their ability to sediment upon ultracentrifugation (Fig. 4B-C), to stimulate kinesin13 ATPase activity (Fig. 4E), and by their filamentous structure observed by negative stained EM (Fig. 4D). Together, these results showed the following: First, tubulin-GTP hydrolysis can be resulted from paclitaxel binding and its effect on tubulin conformation. Second, tubulin-GTP hydrolysis is not an absolute requirement for MT polymerization. Third, paclitaxel-induced conformational change of tubulin dimers, likely straightening as supported by previously published data [57], can facilitate MT polymerization in the absence of tubulin-GTP (and therefore its hydrolysis as well).

Evidence for tubulin-GTP at MT ends - the "GTP Cap". Our finding that conformational change of tubulin dimers, namely its straightening, leads to GTP hydrolysis, prompted us to hypothesize the following: During MT polymerization, as tubulin dimers straighten and incorporate into protofilaments, the conformational change activates the GTPase activity of $\beta$ tubulin. In this scenario, the only tubulin dimers that remain GTP-bound are those at the polymerizing MT ends. This model predicts that during MT polymerization once nucleation has been established, the level of GTP-tubulin at MT ends should remain at similar level as the number of polymerizing ends will stay relatively constant. To test this, we carried out a time course experiment with tubulin in the presence of GTP and $\gamma^{32} \mathrm{P}-\mathrm{GTP}$ tracer, followed by ultracentrifugation-based sedimentation to separate the polymerizing MTs and unpolymerized tubulin dimers. Indeed, we observed that while the net hydrolysis of GTP increased with time as more and more polymers formed (Fig. 4F, left), the amount of GTP in the polymers (i.e. pellets in sedimentation assay) was constant throughout the polymerization process (comparing 5, 10, 20 min time points in Fig. 4F, right). For comparison, we also performed the same experiment with paclitaxel. As expected, paclitaxel eliminated GTP incorporation at MT ends (Fig. 4J). We also observed that the overall GTP hydrolysis in the presence of paclitaxel was lower than that of polymerization enhanced by DMSO, where growing and shrinking of MTs occurred more readily and continuously (Fig. 4G-I). This result is consistent with the understanding that paclitaxel favors MT polymerization, but suppresses its dynamics (and hence lower overall GTP 
1 hydrolysis, marked by lower inorganic phosphate $\left(\mathrm{P}_{\mathrm{i}}\right)$ release than the corresponding DMSO

2 polymerization at all time points, Fig. 4I).

Results from our polymerization experiments predicted that if we started with more

4 polymerization nuclei or smaller/shorter nuclei (and therefore more nuclei at the same concentration), we should observe more GTP incorporation at MT ends. To test this prediction, we prepared shorter and longer seeds by polymerizing GMPCPP-tubulin at two different concentrations. The experimental scheme is illustrated in Fig. 5A (and in Fig. S9A). Higher tubulin concentration favored more numerous spontaneous nucleation and therefore produced shorter seeds (R1, Fig. 5A). Conversely, lower concentration lead to longer MT seeds (R2, Fig. 5A). Alternatively, if we took longer seeds and then sheered them into shorter and more numerous ones with a 25-gauge syringe needle, we would produce seeds of different lengths depending on the number of strokes applied to the seed sample (R2'5x \& R2'10x in Fig. 5A). Using these different seeds, we set up polymerization reactions in the presence of the same concentration of free tubulin-GTP dimers at $5 \mu \mathrm{M}$ in the presence of a radioactive $\gamma^{32} \mathrm{P}-\mathrm{GTP}$ tracer. While all reactions proceeded efficiently (as evidenced by almost complete polymerization shown in sedimentation assay, Fig. 5E-F \& Fig. S9B-C), we consistently observed more $\gamma^{32} \mathrm{P}-\mathrm{GTP}$ incorporations into MT polymers (pellet) in reactions containing more numerous seeds (i.e. more MT ends) while overall GTP hydrolysis did not differ as much among all reactions (Fig. 5C-D \& Fig. S9D-E). This result indicated that overall polymerization did not vary much among reactions since the same concentration of free GTP-tubulin dimers were used in all reactions (Fig. 5C \& S9C). On the other hand, the number of polymerizing ends extending from the seeds varies greatly depending on what type of seeds were used in the reactions, and as a result we observed more GTP incorporation into the polymers in reactions with more numerous polymerizing ends (R1 vs R1', R2 and R2' in Fig. 5B-F \& Fig. S9D).

To provide a quantitative account of the polymers that forms in these reactions, we determined the length of MTs by electron microscopy (EM) (Fig. 5G). We prepared the samples using two methods: one was to dilute the polymerized samples into paclitaxel-containing stabilizing buffer and the other was to fix it with formaldehyde, and then observed them by negative stained EM. The length measurements we obtained were consistent with what we observed in the radioactive tubulin-GTP polymerization experiments: with shorter seeds 
1 produced shorter MTs and longer seeds produced longer MTs. From these measurements and the

2 quantification of GTP incorporation at MT ends (Fig. 5D,F \& Fig. 5H), we could extrapolate the average length of GTP-tubulin-bearing protofilaments at each MT ends ( = average MT length $x$ $\%$ GTP in pellet, corrected by \% tubulin polymerized) . Based on these calculations, we determined the average length of MT ends containing GTP-tubulin to be $69.42 \pm 25.53 \mathrm{~nm}$ (assuming 13 protofilaments/MT). This is in agreement with the length of curved protofilaments at MT ends (40-80 nm), as recently measured by McIntosh et al. [22] using cryo-EM images of MT polymerized in vitro and in various cell types. Together, these data are consistent with the idea that GTP- $\beta$-tubulin-containing MT ends reflect those dimers that have not undergone straightening and therefore still retain their conformational curvature as that of the free dimers.

A model for conformational change driven tubulin-GTP hydrolysis. Based on the experimental data that presented thus far, we propose the follow model for conformational change driven tubulin-GTP hydrolysis (as depicted in Fig. 6). Free GTP-tubulin dimers exist in a native slightly curved state. When they undergo polymerization, tubulin dimers straighten up to incorporate into protofilaments within the microtubule lattice. The straightening process triggers GTP hydrolysis (as evidenced in Fig. 4). The polymer ends represent the transition state of this process and may retain the GTP-bound state prior to straightening (Fig. 4F \& Fig. 5). This transition zone may represent the so-called "GTP cap" described in the literature. As tubulin dimers, free from or at MT ends, encounter kinesin-13 molecules, the complex formation further bends the tubulin dimers into an even more curved conformation, inferred from our published structural study [49]. Our experimental data show that this bending per se does not trigger GTP hydrolysis, since no hydrolysis occurs in the presence of AMP-PNP-kinesin-13s (Fig. 1). However, GTP hydrolysis occurs upon the release of tubulin dimers from the complex (Fig. 1-2), indicating that the transition from the extremely curved state to the less curved native state triggers GTP hydrolysis, even for two less readily hydrolysable GTP analogs (GTP $\gamma \mathrm{S}$ and GMP-

27 CPP). On the other hand, kinesin-13-induced MT depolymerization differs from

28 depolymerization mediated by $\mathrm{Ca}^{2+}$ or cold temperature (Fig. 2). The later process causes the conformational transition of tubulin from straight to the native relaxed curve, which does not 
1 hydrolysis only occurs when tubulin dimer transitions from curved to straighter conformations

2 (Fig. 6, from right to left), but not from straight to curved (Fig. 6, from left to right). In addition,

3 tubulin-GTP hydrolysis does not occur because the tubulin dimer is in a specific conformation,

4 but it is due to the directional structural transition/change between states.

5

\section{DISCUSSION}

Following our previous structural study [49], we report here that Kinesin-13 proteins induced conformational transition of associated tubulin dimers can trigger $\beta$-tubulin GTP turnover. The observation that hydrolysis of one ATP molecule can lead to hydrolysis of two GTP molecules implies that the two tubulin dimers bound to the depolymerase are both in a conformation that is more curved than the native relaxed state of free $\alpha \beta$-tubulin dimers and can increase their GTPase activity upon release. On the other hand, different nucleotide-bound states of $\beta$-tubulin can stimulate kinesin-13 ATPase rate to a different degree, with GTP or its mimic GTP $\gamma$ S-bound being the highest, followed by GDP- and Apo-state (from straighter to more curved conformations, an assessment based on the available published structural information, [54-56] (Fig. S7A-B). We also observed corresponding differences in binding affinity between kinesin-13 proteins and tubulin in different nucleotide states (Fig. S7C-E). By inference from this model, the kinesin-13 ATPase activity is highest when the depolymerase is interacting with tubulin in the shaft of a MT, as the tubulin dimers there are straightest of all and in tandem arrays. This interaction does not, however, lead to depolymerization. At MT ends, kinesin-13 proteins can interact with tubulin dimers on the exposed protofilament ends leading to the bending of tubulin dimers and spraying outward of protofilaments to facilitate its depolymerization. This is accompanied by the hydrolysis of tubulin-GTP dimers at MT ends upon their dissociation from kinesin-13 molecules, leading to the removal of GTP-tubulin-containing protofilament tips. Results form our experiments with the use of MTs formed by slowly hydrolyzable GTP analogs (GTP $\gamma$ S and GMPCPP, in Fig. 2A-C, Fig. S4A-C \& Fig. S5A-C), support this assertion.

What we did not expect in the beginning was that our investigation on kinesin-13 mediated MT depolymerization would reveal something more fundamental, namely the molecular basis of $\beta$-tubulin-GTP hydrolysis. It is generally thought that GTP hydrolysis leads to a conformational change of tubulin dimers as they incorporate into MT polymer [16, 23, 58]. Our data suggest otherwise. Our experiments with paclitaxel showed that it is the change of tubulin 
1 conformation that triggers GTP hydrolysis (Fig. 4). In addition, our data on tubulin-GTP with

2 AMP-PNP-bound kinesin-13s and DARPin also indicate that it is the change/transition of conformation, and not a particular nucleotide-bound state, that facilitates the GTP hydrolysis

4 (Fig. 1-2). Furthermore, this change needs to be directional (from a more curved to a less curved

5 conformation, but not vice versa) to be productive, as we have shown the lack of GTP turnover with calcium or cold induced depolymerization (from a straight to a more curved conformation).

7 It also appears that the degree of structural change matters too. For example, GTP $\gamma \mathrm{S}$ - and

8 GMPCPP-bound tubulins, unlike GTP-tubulins do not undergo hydrolysis during polymerization.

9 There are at least two compatible explanations for that. One is that GTP $\gamma \mathrm{S}$ and GMPCPP are less readily hydrolyzed than GTP. Another is that GTP $\gamma$ S- and GMPCPP-bound tubulins are in a

11 straighter conformation than GTP-tubulin, as suggested by published structural data [23, 30, 31,

12 59]. As a result, their structural changes upon polymerization are not as great as that of GTP-

13 tubulin and therefore not enough to trigger hydrolysis. We favor this explanation because

14 structural change can indeed be severe enough to induce hydrolysis of GTP $\gamma \mathrm{S}$ and GMPCPP, as

15 we have seen this occurring in KIF2A-NM induced MT depolymerization (Fig 2A-C \& Fig. S5A-C). Together, our data strongly support conformational change-induced tubulin-GTP

17 hydrolysis.

Our data from in vitro MT polymerization provide a quantitative measurement on the length of the elusive "GTP cap". Our conformational-based model suggests that this length is directly linked to the curvature of the tubulin dimers. It implies that this cap should reflect the outwardly tapering curvature of protofilament ends that has been commonly observed in previous EM studies [21-23]. Based on our calculation, the length of the "GTP cap" is about 70 $\mathrm{nm}$, at least for MT polymerized in vitro. This is consistent with a recent study by McIntosh et al. which determined the length of the curved protofilament ends to be about $40-80 \mathrm{~nm}$, both in vitro and in multiple cell types, ranging from yeast to human [22]. The agreement between these two measurements suggest that the curved tubulin-GTP dimers are added to the MT ends during

27 polymerization and the subsequent straightening of the newly added subunits, in part by forming lateral bond with adjacent protofilaments, activates $\beta$-tubulin-GTPase activity to accelerate GTP turnover. Since nucleotide hydrolysis is a transitional process and not instantaneous, it is possible that there are still some tubulin dimers in their unhydrolyzed GTP or GDP-Pi states near the ends 
1 of the polymerizing ends of MTs, as have been visualized indirectly by the use of fluorescently

2 labelled EB1 [36, 60].

The model that we put forth here is incompatible with the idea of GTP islands that exist

4 deep in the middle of MT lattice as those tubulin dimers should be already in the straight

5 conformation and therefore should be in the GDP-bound state. However, our model is not in

6 conflict with the idea that damage can occur in the MT middle and that the transient existence of

7 GTP-containing dimers during the repair of damaged protofilaments in the MT lattice [61].

8 Beside this special scenario, tubulin-GTP dimers should be restricted to the extremities on MT

9 polymers, at least in vitro. Taking this a step further, our model implies another provocative idea:

10 the existence of the GTP cap may be merely a transition state of the tubulin polymerization

11 process; the "GTP cap" simply marks the polymerizing ends, but itself per se does not offer

12 active stabilizing role besides keeping the tubulin-GDP lattice from being exposed. In cells,

13 however, MT end-binding proteins such as EB1 and its associated factors (e.g. the kinesin-13

14 KIF2C, KIF18B, and others) can modulate MT polymerization dynamics spatially and

15 temporally to regulate different cellular processes throughout the cell cycle. It is conceivable that

16 these proteins may interact with tubulin dimers at MT ends and alter their conformations, and

17 thereby modulating the extent of the GTP caps in cells. It is also conceivable that some MAPs

18 (e.g. MT repair MAPs or tubulin modifying enzymes) could keep sections of MT middle in

19 GTP-bound form, creating the apparent "GTP" islands observed in cells to serve specific cellular

20 functions [26]. All in all, the intriguing ability for $\alpha \beta$-tubulin dimers to self-organize into

21 polymers of hollow tubules, the underlying molecular mechanisms that drive this process, and

22 how associated factors regulate MT dynamics in space and time throughout the cell cycle will

23 continue to fascinate us for years to come. 
Materials: Tubulin, DARPin and Kinesin-13 protein constructs were prepared as previously described [49, 62]. Most biochemicals were purchased from Bioshop Canada Inc. Nucleotides were obtained from Jena Bioscience via Cedarlane (Ontario, Canada). GDP detection kit was from ProFoldin (MA, USA) and ADP-Glo ${ }^{\mathrm{TM}}$ Kinase Assay kit from Promega Corporation. Radio-labeled nucleotides ( $\left.\alpha{ }^{32} \mathrm{P}-\mathrm{ATP}, \gamma^{32} \mathrm{P}-\mathrm{GTP},{ }^{35} \mathrm{~S}-\mathrm{GTP} \gamma \mathrm{S}\right)$ were purchased from Perkin Elmer.

\section{Microtubule Polymerization}

DMSO polymerization: A premix was prepared with 2X BRB80, 2 mM DTT, 2 mM GTP or GTP $\gamma$ S and 20\% DMSO. Recycled tubulin was thawed on ice and then an equal volume of the premix was added to it. Polymerization was done by incubating the reaction mixture in a circulating precision water bath at $37^{\circ} \mathrm{C}$ for $25-30 \mathrm{~min}$.

We pelleted the ${ }^{35} \mathrm{~S}$-labeled GTP $\gamma \mathrm{S}$ MTs through $1 \mathrm{ml}$ of warm $40 \%$ glycerol in BRB80 cushion by spinning down at 90K RPM in a Sorvall TLA100 rotor for 5 min and then resuspended the MTs in BRB80 buffer containing paclitaxel.

Taxol polymerization: Paclitaxel polymerization reaction mixture was prepared with $10 \mu \mathrm{M}$ recycled tubulin in the presence of $200 \mu \mathrm{M}$ GDP, $20 \mu \mathrm{M}$ of paclitaxel and $1 \mathrm{mM}$ DTT in $1 \mathrm{X}$ BRB80. Polymerization was done as described above.

Polymerization from GMPCPP seeds: GMPCPP seeds were prepared as follows: Recycled tubulin was thawed on ice and diluted to $30 \mu \mathrm{M}$ in 1X BRB80 with $1 \mathrm{mM}$ DTT and $0.2 \mathrm{mM}$ GMPCPP. The mixture was incubated on ice for $10 \mathrm{~min}$ and then clarified by spinning down at 90K RPM in a Sorvall TLA100 rotor for $5 \mathrm{~min}$ at $2^{\circ} \mathrm{C}$. The supernatant was recovered, aliquoted to $5 \mu \mathrm{l}$ and snap-frozen in liquid nitrogen and stored at $-80^{\circ} \mathrm{C}$ for later usage. Short GMPCPP seeds were prepared by incubating the aliquoted $30 \mu \mathrm{M}$ GMPCPP-bound tubulin mixture at $37^{\circ} \mathrm{C}$ in a precision circulating water bath for 15-20 min. The mix was then diluted to $100 \mu \mathrm{l}$ using warm BRB80 + $1 \mathrm{mM}$ DTT and spun down at 90K RPM for 5 min at $25^{\circ} \mathrm{C}$ using TLA100 rotor. The supernatant was discarded, and the pellet was re-suspended in $100 \mu \mathrm{l}$ of warm BRB80 with $1 \mathrm{mM}$ DTT. To prepare long GMPCPP seeds, the aliquoted $30 \mu \mathrm{M}$ GMPCPP-bound tubulin 
mixture was diluted to $3 \mu \mathrm{M}$ in warm $1 \mathrm{X}$ BRB $80+1 \mathrm{mM}$ DTT before incubating at $37^{\circ} \mathrm{C}$ for 15 $20 \mathrm{~min}$. After preparation, the long seeds could be sheared into shorter seeds by stroking the solution through a 25 -gauge needle for 5 or 10 times. To polymerize microtubules from GMPCPP seeds, the prepared seeds (short/long/sheared) were added at either 1 or $0.25 \mu \mathrm{M}$ to a polymerization mix containing $5 \mu \mathrm{M}$ recycled tubulin and $200 \mu \mathrm{M}$ GTP and incubated at $37^{\circ} \mathrm{C}$

6 for 25-30 min as described above.

\section{Tubulin / Kinesin-13 mediated nucleotide hydrolysis assays}

Kinesin-13 mediated MT depolymerization or tubulin-GTP hydrolysis assays were carried out as previously described $[49,62]$. Briefly, reactions were assembled in $30 \mu$ volume in a BRB80based buffer (80 mM PIPES, pH 6.8, 1 mM MgCl $2,1 \mathrm{mM}$ EGTA, $75 \mathrm{mM} \mathrm{KCl}, 0.25 \mathrm{mg} / \mathrm{mL}$ bovine serum albumin, $1 \mathrm{mM}$ DTT, and $0.02 \%$ Tween), with the indicated concentrations of tubulin dimers, microtubules, kinesin-13 protein constructs, DARPin, and nucleotides. Reactions were carried out at room temperature for the specified lengths of time. Cold- and calciuminduced MT depolymerizations were performed in a similar manner in the absence of kinesin-13 protein constructs. After the reactions (in the case of microtubule polymerization and depolymerization assays), MT polymers remaining were separated from the free tubulin dimers by an ultracentrifugation-based sedimentation in a Sorvall TLA100 rotor at 80K RPM for 5 min at $25^{\circ} \mathrm{C}$. The supernatant fractions were retrieved from the sedimentation mixture and added to $1 / 4$ volume of $4 \times$ Laemmli buffer. The polymer-containing pellets were re-suspended in an equal volume of hot $1 \times$ Laemmli buffer (prepared by diluting the $4 \mathrm{x}$ buffer in depolymerization reaction buffer). Equal portions of the supernatant and pellet samples were resolved on SDSPAGE. The gel was stained with Coomassie blue dye and scanned with either Epson Perfection 4990 Photo or CanoScan 5600F digital scanners. The bands were quantified using ImageJ (NIH).

\section{Nucleotide hydrolysis detection methods}

27 Malachite green phosphate detection assay: Malachite green-based phosphate detection assay was used to measure ATP and GTP hydrolysis rate, as previously described [62]. Briefly, reactions were assembled in $30 \mu \mathrm{l}$ volume in the same BRB80-based buffer used for the nucleotide hydrolysis assay, with the indicated concentrations of tubulin/microtubules, kinesin13 protein constructs, DARPin, and nucleotides. Reactions were allowed to proceed for the 
1 indicated lengths of time (10-15 $\mathrm{min})$. The reactions were then quenched using equal volume (30

$2 \mu \mathrm{l}$ ) of $90 \mathrm{mM}$ perchloric acid. We then added $30 \mu \mathrm{l}$ of the quenched mixture to $40 \mu \mathrm{l}$ malachite

3 green reagent in a 384-well transparent plate to develop the color. After 5-10 min of incubation

4 at room temperature, the level of phosphate generated in each well was quantified by measuring

5 the absorbance at $620 \mathrm{~nm}$ using a TECAN infinite M200 PRO plate reader. A standardized

6 calibration curve was generated using a titration of monobasic potassium phosphate $\left(\mathrm{KH}_{2} \mathrm{PO}_{4}\right)$ in

7 the same reaction buffer (Fig. S10A).

$$
A b s=a[P]+b
$$

And the equation was used to calculate the concentration of phosphate generated in each well.

$$
[P]=\frac{A b s-b}{a}
$$

11 Verification of signal (or the lack thereof) from the presence of thiophosphate was done in

12 similar manner with a titration of thiophosphate in the same reaction buffer (Fig. S10B). This

13 level of background thiophosphate signal was used for background subtraction whenever

14 applicable.

GDP detection assay: MicroMolar GDP assay kit (ProFoldin) was used to measure the level of GTP hydrolysis. A premix solution was prepared according to manufacturer instructions in a Tris- $\mathrm{HCl}$ based buffer (50 mM Tris- $\mathrm{HCl}$, pH 8.0, $3 \mathrm{mM} \mathrm{MgCl}_{2}, 0.2 \mathrm{mM}$ EDTA, $0.5 \mathrm{mM}$ DTT, $50 \mathrm{mM} \mathrm{NaCl}, 0.003 \%$ Brij-35). Equal volumes (15 $\mu \mathrm{l})$ of reaction samples and premix solution were mixed and incubated for $45 \mathrm{~min}$ at room temperature. Then $30 \mu \mathrm{l}$ of $1 \mathrm{X}$ Fluorescent dye was added to the mixture and after 5 min the fluorescence intensity was read at $535 \mathrm{~nm}$ with

22 excitation at $485 \mathrm{~nm}$ using TECAN infinite M200 PRO plate reader. GDP samples of known concentrations were used to obtain a linear standard curve of the fluorescent intensity $(\mathrm{Fc})$ values and the GDP concentration [GDP] (Fig. S1A).

$$
F c=a[G D P]+b
$$

27 and $b$ values from the standard curve.

$$
[G D P]=\frac{F c-b}{a}
$$


1 To verify the level of signal interference from the presence of ADP in the mixture, another standard curve was plotted from a separate set of assays using a titration of known ADP concentrations (Fig. S1B).

4

$5 \quad$ ADP Glo assay: Reactions were assembled in $25 \mu 1$ total volume, as described for the Malachite Green assay. Once the reactions were complete, the ADP-Glo ${ }^{\mathrm{TM}}$ Assay was performed in two steps. The reaction mixture was then added to $25 \mu 1$ of ADP-Glo ${ }^{\mathrm{TM}}$ Reagent and incubated at

8 room temperature for 40 minutes. Then the mixture was added to $50 \mu 1$ of Kinase Detection reagent (to convert ADP to ATP and introduce luciferase and luciferin to detect ATP) and incubated at room temperature for 40-60 minutes (based on the concentration of ATP). The mixture was added to a 96-well white solid bottom plate in duplicates and luminescence was measured using TECAN infinite M200 PRO plate reader. Four ATP-to-ADP conversion standard curves were prepared at different ATP+ADP concentrations $(1,10,100$ or $1000 \mu \mathrm{M})$, in $25 \mu 1$ of $1 \mathrm{X}$ reaction buffer. ADP-Glo ${ }^{\mathrm{TM}}$ Kinase Assays were performed at room temperature as described for the samples and four standard curves were plotted from the luminescence values of the different concentrations of ATP/ADP (Fig. S11).

Radioactivity assay: Nucleotide hydrolysis or MT polymerization assays were performed in the same buffers as described, except that the reaction mixtures were supplemented with $\alpha^{32} \mathrm{P}-\mathrm{ATP}$, $\gamma^{32} \mathrm{P}-\mathrm{GTP},{ }^{35} \mathrm{~S}-\mathrm{GTP} \gamma \mathrm{S}$ tracers for the detection of hydrolysis. After the specified incubation time, reactions were quenched by the addition of equal volume of $1 \mathrm{~N}$ formic acid. Small fractions of the samples were removed and spotted on a poly(ethyleneimine)-cellulose plate (EMD Millipore - TLC PEI Cellulose F - 1.05579.0001) and let air-dried for 5-10 min. Samples on the plate were resolved by thin-layer chromatography (TLC) in a glass chamber using $0.375 \mathrm{M} \mathrm{KH}_{2} \mathrm{PO} 4$ (pH 3.5) as a resolving buffer. Afterwards, the TLC plate was air dried for at least 1 hour before exposure to an X-ray film or a phosphor-imager screen. The radioactive spots on the developed X-ray films or on the scanned phospho-imager screens were quantified using ImageJ software or Typhoon FLA 9500 laser scanner, respectively. The level of hydrolysis was calculated using the ratio of the hydrolyzed spot and the initial concentration of the nucleotides $\left(\mathrm{ATP}_{\text {initial }}\right.$ or $\mathrm{GTP}_{\text {initial }}$ 30 in the solution).

$$
[P i]=\left[G T P_{\text {initial }}\right] \times(P i /(P i+G T P)
$$


or

$$
[A D P]=\left[A T P_{\text {initial }}\right] \times(A D P /(A D P+A T P)
$$

4 Tubulin-Kinesin-13 Binding Assay

5 Tubulin-kinesin-13 binding assay was carried out via immunoprecipitation using Ni-charged MagBeads (GenScript, NJ), to pull down Histidine-tagged kinesin-13 proteins. Briefly, magnetic beads were washed with BRB80-based binding buffer (80 mM PIPES, pH 6.8, $1 \mathrm{mM} \mathrm{MgCl}, 1$ mM EGTA, $75 \mathrm{mM} \mathrm{KCl}, 0.25 \mathrm{mg} / \mathrm{mL}$ bovine serum albumin, $1 \mathrm{mM} \mathrm{DTT}$, and $0.02 \%$ Tween). The reactions were started by adding kinesin-13 proteins at the indicated concentrations to $2 \mu \mathrm{l}$ of magnetic beads in BRB80-based binding buffer. The indicated concentrations of tubulin dimers and nucleotides were then added to the reaction mixtures that were assembled in a final volume of $30 \mu \mathrm{l}$. Reactions were carried out at room temperature for 5 minutes. The beads were then pulled down using a magnetic stand. The supernatant fractions were retrieved from the sedimentation mixture and added to $1 / 4$ volume of $4 \times$ Laemmli buffer. The beads were resuspended in an equal volume of hot $1 \times$ Laemmli buffer (prepared by diluting the $4 \mathrm{x}$ buffer in the binding reaction buffer). Equal portions of the supernatant and pellet samples were resolved on SDS-PAGE. The gel was stained with Coomassie blue dye and scanned with either Epson Perfection 4990 Photo or CanoScan 5600F digital scanners. The bands were quantified using ImageJ (NIH).

\section{Negative staining of MTs by Transmission Electron Microscopy (TEM)} $\mathrm{Ni}$; Electron Microscopy Sciences) were glow discharged via a Pelco easy glow (Pelco, Fresno, USA) for 30 seconds. MT samples were prepared as described above, just right before grid preparation and were diluted to $0.5 \mu \mathrm{M}$. $10 \mu \mathrm{l}$ of the diluted sample was placed on a piece of parafilm and grid was placed on the top (with carbon side touching the sample) for $\sim 1$ min. Excess liquid was then removed by gently tilting the grid sideway on a Whatman blotting paper. The grid was then rinsed with a droplet of $\mathrm{dH}_{2} \mathrm{O}$ and blotted again with a fresh Whatman paper. The rinse procedure was repeated two more times and the samples on the grid were then stained with $10 \mu \mathrm{l}$ of $0.5 \%$ filtered EM-grade Uranyl Acetate (UA). After removing the excess UA by blotting, the grid was air dried for at least 1 hour. Grids were examined at the EM facility at the 
bioRxiv preprint doi: https://doi.org/10.1101/2020.09.08.288019; this version posted September 10, 2020. The copyright holder for this preprint (which was not certified by peer review) is the author/funder. All rights reserved. No reuse allowed without permission.

1 Université de Montréal in a FEI Tecnai 12 (Eindhoven, The Netherlands) transmission electron

2 microscope operating at $80 \mathrm{kV}$. For each experimental condition, the lengths of 300 microtubules

3 were quantified using ImageJ (NIH).

4 
1 Acknowledgement: We thank the LSCTB platform at the Université de Montréal for the access

2 to the Electron Microscopy facility. We thank members of the Kwok lab for their inputs and

3 suggestions, and Drs. Richard J McIntosh and Nikita Gudimchuk for their critical reviews on the

4 manuscript. We acknowledge the funding support from the Canadian Institutes of Health

5 Research (to B.H.K: PJT 148982 \& 152920). B.H.K. is a recipient of the Fonds de recherche du

6 Québec - Santé (FRQS) Chercheure-boursière Junior 1 and Junior 2 Awards and the Canadian

7 Institutes of Health Research (CIHR) New Investigator Award. M.P. is supported by a FRQS

8 doctoral fellowship and by doctoral and financial programs at the Université de Montréal.

Author information: Correspondence and requests for materials should be addressed to

11 benjamin.kwok@umontreal.ca.

Author contributions: B.H.K. conceived the project. M.P. and B.H.K. designed and performed experiments. M.P. and B.H.K analyzed and interpreted data. B.H.K. wrote the first draft. M.P. and B.H.K. prepared and completed the manuscript.

17 Declaration of Interests: The authors declare no competing financial interests. 


\section{REFERENCES}

1. Desai, A., and Mitchison, T.J. (1997). MICROTUBULE POLYMERIZATION DYNAMICS. Annual Review of Cell and Developmental Biology 13, 83-117.

2. Erickson, H.P., and Pantaloni, D. (1981). The role of subunit entropy in cooperative assembly. Nucleation of microtubules and other two-dimensional polymers. Biophysical Journal 34, 293309.

3. Moritz, M., Braunfeld, M.B., Sedat, J.W., Alberts, B., and Agard, D.A. (1995). Microtubule nucleation by $\gamma$-tubulin-containing rings in the centrosome. Nature 378, 638-640.

4. Osawa, F., and Asakura, S. (1975). Thermodynamics of the polymerization of protein, (London; New York: Academic Press).

5. Mitchison, T., and Kirschner, M. (1984). Dynamic instability of microtubule growth. Nature 312, 237-242.

6. Arai, T., and Kaziro, Y. (1977). Role of GTP in the Assembly of Microtubules. The Journal of Biochemistry 82, 1063-1071.

7. Caplow, M., and Shanks, J. (1990). Mechanism of the microtubule GTPase reaction. Journal of Biological Chemistry 265, 8935-8941.

8. Stewart, R.J., Farrell, K.W., and Wilson, L. (1990). Role of GTP hydrolysis in microtubule polymerization: evidence for a coupled hydrolysis mechanism. Biochemistry 29, 6489-6498.

9. David-Pfeuty, T., Erickson, H.P., and Pantaloni, D. (1977). Guanosinetriphosphatase activity of tubulin associated with microtubule assembly. Proceedings of the National Academy of Sciences of the United States of America 74, 5372-5376.

10. MacNeal, R.K., and Purich, D.L. (1978). Stoichiometry and role of GTP hydrolysis in bovine neurotubule assembly. J Biol Chem 253, 4683-4687.

11. Spiegelman, B.M., Penningroth, S.M., and Kirschner, M.W. (1977). Turnover of tubulin and the N site GTP in chinese hamster ovary cells. Cell 12, 587-600.

12. Carlier, M.F., and Pantaloni, D. (1981). Kinetic analysis of guanosine 5'-triphosphate hydrolysis associated with tubulin polymerization. Biochemistry 20, 1918-1924.

13. Weisenberg, R., Deery, W., and Dickinson, P. (1976). Tubulin-nucleotide interactions during the polymerization and depolymerization of microtubules. Biochemistry 15, 4248-4254.

14. Bowne-Anderson, H., Zanic, M., Kauer, M., and Howard, J. (2013). Microtubule dynamic instability: a new model with coupled GTP hydrolysis and multistep catastrophe. BioEssays : news and reviews in molecular, cellular and developmental biology 35, 452-461.

15. Mandelkow, E.M., Mandelkow, E., and Milligan, R.A. (1991). Microtubule dynamics and microtubule caps: a time-resolved cryo-electron microscopy study. Journal of Cell Biology 114, 977-991.

16. Alushin, G.M., Lander, G.C., Kellogg, E.H., Zhang, R., Baker, D., and Nogales, E. (2014). Highresolution microtubule structures reveal the structural transitions in $\alpha \beta$-tubulin upon GTP hydrolysis. Cell 157, 1117-1129.

17. Grafmüller, A., Noya, E.G., and Voth, G.A. (2013). Nucleotide-dependent lateral and longitudinal interactions in microtubules. J Mol Biol 425, 2232-2246.

18. Buey, R.M., Diaz, J.F., and Andreu, J.M. (2006). The Nucleotide Switch of Tubulin and Microtubule Assembly: A Polymerization-Driven Structural Change. Biochemistry 45, 5933-5938.

19. Nawrotek, A., Knossow, M., and Gigant, B.t. (2011). The Determinants That Govern Microtubule Assembly from the Atomic Structure of GTP-Tubulin. Journal of Molecular Biology 412, 35-42.

20. Rice, L.M., Montabana, E.A., and Agard, D.A. (2008). The lattice as allosteric effector: structural studies of alphabeta- and gamma-tubulin clarify the role of GTP in microtubule assembly. 
Proceedings of the National Academy of Sciences of the United States of America 105, 53785383.

21. Chrétien, D., Fuller, S.D., and Karsenti, E. (1995). Structure of growing microtubule ends: twodimensional sheets close into tubes at variable rates. Journal of Cell Biology 129, 1311-1328.

22. McIntosh, J.R., Oâ€ ${ }^{\mathrm{TM} T o o l e, ~ E ., ~ M o r g a n, ~ G ., ~ A u s t i n, ~ J ., ~ U l y a n o v, ~ E ., ~ A t a u l l a k h a n o v, ~ F ., ~ a n d ~}$ Gudimchuk, N. (2018). Microtubules grow by the addition of bent guanosine triphosphate tubulin to the tips of curved protofilaments. Journal of Cell Biology 217, 2691-2708.

23. Müller-Reichert, T., Chrétien, D., Severin, F., and Hyman, A.A. (1998). Structural changes at microtubule ends accompanying GTP hydrolysis: information from a slowly hydrolyzable analogue of GTP, guanylyl (alpha, beta)methylenediphosphonate. Proceedings of the National Academy of Sciences of the United States of America 95, 3661-3666.

24. Prota, A.E., Bargsten, K., Zurwerra, D., Field, J.J., DÃaz, J.F., Altmann, K.-H., and Steinmetz, M.O. (2013). Molecular Mechanism of Action of Microtubule-Stabilizing Anticancer Agents. Science 339, 587-590.

25. Carlier, M.-F. (1982). Guanosine-5'-triphosphate hydrolysis and tubulin polymerization. Molecular and Cellular Biochemistry 47, 97-113.

26. Dimitrov, A., Quesnoit, M.I., Moutel, S., Cantaloube, I., PoÃ $1 / 45$, C., and Perez, F. (2008). Detection of GTP-Tubulin Conformation in Vivo Reveals a Role for GTP Remnants in Microtubule Rescues. Science 322, 1353-1356.

27. Cassimeris, L. (2009). Stathmin regulates centrosomal nucleation of microtubules and tubulin dimer/polymer partitioning. . Molecular Biolology of the Cell 20.

28. Caplow, M. (1992). Microtubule dynamics. Current Opinion in Cell Biology 4, 58-65.

29. Erickson, H.P., and O'Brien, E.T. (1992). Microtubule Dynamic Instability and GTP Hydrolysis. Annual Review of Biophysics and Biomolecular Structure 21, 145-166.

30. Maurer, S.P., Bieling, P., Cope, J., Hoenger, A., and Surrey, T. (2011). GTP $\gamma S$ microtubules mimic the growing microtubule end structure recognized by end-binding proteins (EBs). Proceedings of the National Academy of Sciences 108, 3988-3993.

31. Maurer, SebastianÂ P., Fourniol, FranckÂ J., Bohner, G., Moores, CarolynÂA A., and Surrey, T. (2012). EBs Recognize a Nucleotide-Dependent Structural Cap at Growing Microtubule Ends. Cell 149, 371-382.

32. Zhang, R., Alushin, GregoryÂ M., Brown, A., and Nogales, E. (2015). Mechanistic Origin of Microtubule Dynamic Instability and Its Modulation by EB Proteins. Cell 162, 849-859.

33. Zanic, M., Stear, J.H., Hyman, A.A., and Howard, J. (2009). EB1 recognizes the nucleotide state of tubulin in the microtubule lattice. PLoS ONE 4, e7585.

34. Brouhard, G., and Sept, D. (2012). Microtubules: Sizing Up the GTP Cap. Current Biology 22, R802-R803.

35. Seetapun, D., Castle, BrianÂ T., Mclntyre, AlistairÂ J., Tran, PhongÂ T., and Odde, DavidÂ J. (2012). Estimating the Microtubule GTP Cap Size InÂ Vivo. Current Biology 22, 1681-1687.

36. Bieling, P., Laan, L., Schek, H., Munteanu, E.L., Sandblad, L., Dogterom, M., Brunner, D., and Surrey, T. (2007). Reconstitution of a microtubule plus-end tracking system in vitro. Nature 450, 1100-1105.

37. Duellberg, C., Cade, N.I., Holmes, D., and Surrey, T. (2016). The size of the EB cap determines instantaneous microtubule stability. eLife 5, e13470

C13471 - eLife 12016;13475:e13470.

38. Westermann, S., and Weber, K. (2003). Post-translational modifications regulate microtubule function. Nature Reviews Molecular Cell Biology 4, 938-948.

39. Wloga, D., and Gaertig, J. (2010). Post-translational modifications of microtubules. Journal of Cell Science 123, 3447-3455. 
40. Ayaz, P., Ye, X., Huddleston, P., Brautigam, C.A., and Rice, L.M. (2012). A TOG:Ît $\hat{l}^{2}$-tubulin Complex Structure Reveals Conformation-Based Mechanisms for a Microtubule Polymerase. Science 337, 857-860.

41. Roostalu, J., Cade, N.I., and Surrey, T. (2015). Complementary activities of TPX2 and chTOG constitute an efficient importin-regulated microtubuleÂ nucleation module. Nature Cell Biology 17, 1422-1434.

42. Gupta, M.L., Carvalho, P., Roof, D.M., and Pellman, D. (2006). Plus end-specific depolymerase activity of Kip3, a kinesin-8 protein, explains its role in positioning the yeast mitotic spindle. Nature Cell Biology 8, 913-923.

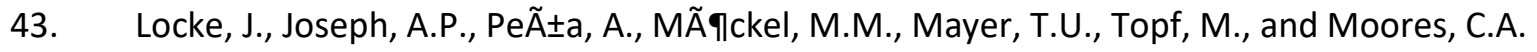
(2017). Structural basis of human kinesin-8 function and inhibition. Proceedings of the National Academy of Sciences 114, E9539-E9548.

44. Savoian, M.S., and Glover, D.M. (2010). <em>Drosophila</em> Klp67A binds prophase kinetochores to subsequently regulate congression and spindle length. Journal of Cell Science 123, 767-776.

45. Howard, J., and Hyman, A.A. (2007). Microtubule polymerases and depolymerases. Curr Opin Cell Biol 19, 31-35.

46. Walczak, C.E., Gayek, S., and Ohi, R. (2013). Microtubule-depolymerizing kinesins. Annu Rev Cell Dev Biol 29, 417-441.

47. Friel, C.T., and Howard, J. (2011). The kinesin-13 MCAK has an unconventional ATPase cycle adapted for microtubule depolymerization. The EMBO Journal 30, 3928-3939.

48. Hunter, A.W., Caplow, M., Coy, D.L., Hancock, W.O., Diez, S., Wordeman, L., and Howard, J. (2003). The Kinesin-Related Protein MCAK Is a Microtubule Depolymerase that Forms an ATPHydrolyzing Complex at Microtubule Ends. Molecular Cell 11, 445-457.

49. Trofimova, D., Paydar, M., Zara, A., Talje, L., Kwok, B.H., and Allingham, J.S. (2018). Ternary complex of Kif2A-bound tandem tubulin heterodimers represents a kinesin-13-mediated microtubule depolymerization reaction intermediate. Nature Communications 9, 2628.

50. Desai, A., Verma, S., Mitchison, T.J., and Walczak, C.E. (1999). Kin I Kinesins Are MicrotubuleDestabilizing Enzymes. Cell 96, 69-78.

51. Noda, Y., Niwa, S., Homma, N., Fukuda, H., Imajo-Ohmi, S., and Hirokawa, N. (2011). Phosphatidylinositol 4-phosphate 5-kinase alpha (PIPKÎt) regulates neuronal microtubule depolymerase kinesin, KIF2A and suppresses elongation of axon branches. Proceedings of the National Academy of Sciences 109, 1725-1730.

52. Cassimeris, L.U., Wadsworth, P., and Salmon, E.D. (1986). Dynamics of microtubule depolymerization in monocytes. J Cell Biol 102, 2023-2032.

53. O'Brien, E.T., Salmon, E.D., and Erickson, H.P. (1997). How calcium causes microtubule depolymerization. Cell motility and the cytoskeleton 36, 125-135.

54. Brouhard, G.J., and Rice, L.M. (2014). The contribution of $\alpha \beta$-tubulin curvature to microtubule dynamics. The Journal of cell biology 207, 323-334.

55. Pecqueur, L., Duellberg, C., Dreier, B., Jiang, Q., Wang, C., Plückthun, A., Surrey, T., Gigant, B., and Knossow, M. (2012). A designed ankyrin repeat protein selected to bind to tubulin caps the microtubule plus end. Proceedings of the National Academy of Sciences of the United States of America 109, 12011-12016.

56. Ravelli, R.B., Gigant, B., Curmi, P.A., Jourdain, I., Lachkar, S., Sobel, A., and Knossow, M. (2004). Insight into tubulin regulation from a complex with colchicine and a stathmin-like domain. Nature 428, 198-202.

57. Elie-Caille, C.I., Severin, F., Helenius, J., Howard, J., Muller, D.J., and Hyman, A.A. (2007). Straight GDP-Tubulin Protofilaments Form in the Presence of Taxol. Current Biology 17, 1765-1770. 
58. Mitchison, T., and Kirschner, M. (1984). Microtubule assembly nucleated by isolated centrosomes. Nature 312, 232-237.

59. Wang, H.-W., and Nogales, E. (2005). Nucleotide-dependent bending flexibility of tubulin regulates microtubule assembly. Nature 435, 911-915.

60. Rickman, J., Duellberg, C., Cade, N.I., Griffin, L.D., and Surrey, T. (2017). Steady-state EB cap size fluctuations are determined by stochastic microtubule growth and maturation. Proceedings of the National Academy of Sciences 114, 3427-3432.

61. Aumeier, C., Schaedel, L., Gaillard, J., John, K., Blanchoin, L., and Théry, M. (2016). Self-repair promotes microtubule rescue. Nature Cell Biology 18, 1054-1064.

62. Talje, L., Ben El Kadhi, K., Atchia, K., Tremblay-Boudreault, T., Carreno, S., and Kwok, B.H. (2014). DHTP is an allosteric inhibitor of the kinesin-13 family of microtubule depolymerases. FEBS Lett $588,2315-2320$. 


\section{FIGURE LENGEND}

\section{Fig. 1 | Binding dynamics of the Kinesin-13 KIF2A with tubulin dimers trigger $\beta$-tubulin-} GTP hydrolysis.

(A) Tubulin-GTP hydrolysis measured by GDP production (ProFoldin GDP detection assay) when tubulin dimers $(4 \mu \mathrm{M})$ and GTP $(0.2 \mathrm{mM})$ were incubated alone, with DARPin or in the presence of KIF2A-NM (200 nM) with AMP-PNP or with AMP-PNP and DARPin for 15 minutes at room temperature. (B) To determine the effect of DARPin, tubulin-GTP hydrolysis measured by GDP production when tubulin-GTP dimers were incubated in the presence of KIF2A-NM and AMP-PNP with different concentrations of DARPin, under the same condition as in (A). (C) Time-course experiments comparing the tubulin-GTP turnovers between tubulin dimers that were incubated in the presence of KIF2A-NM with AMP-PNP and DARPin and those with KIF2A-NM with ATP under the same condition as in (A). For (A-C), the level of GDP was measured using a GDP detection assay (ProFoldin). (D) A time course experiment in which basal tubulin-GTP hydrolysis was measured and compared with in KIF2A-NM-induced GTP hydrolysis (in the presence of ATP) using a $\gamma^{32}$ P-GTP radio-labeled GTPase assay under the same experimental setting as described as in $(\mathrm{C}) \cdot \gamma^{32} \mathrm{P}-\mathrm{GTP}$ and ${ }^{32} \mathrm{P}_{\mathrm{i}}$ were resolved by thin layer chromatography and exposed to a film. A representative autoradiogram is shown. (E) Quantification of ${ }^{32} \mathrm{P}_{\mathrm{i}}$ of each time point in the time course experiment shown in (D). All the data shown represent an average of at least three independent experimental runs. Error bars represent standard deviations. (ns (not significant): $\mathrm{p}>0.05 ; * \mathrm{p} \leq 0.05 ; * * \mathrm{p} \leq 0.01 ; * * * \mathrm{p} \leq 0.001$, by Student's $t$-test)

\section{Fig. 2 | Kinesin-13 mediated MT depolymerization, unlike non-catalytically induced depolymerization, triggers tubulin-GTP hydrolysis.}

(A) MT depolymerization assay was set up using ${ }^{35} \mathrm{~S}-\mathrm{GTP} \gamma \mathrm{S}-$ labeled MTs alone (Ctrl) or in the presence of DARPin alone, with KIF2A-NM and AMP-PNP, with KIF2A-NM, AMP-PNP and DARPin, or with KIF2A-NM and ATP. Reactions were carried out at room temperature for 15 minutes. Samples containing ${ }^{35} \mathrm{~S}-\mathrm{GTP} \gamma \mathrm{S}$ and ${ }^{35} \mathrm{~S}-\mathrm{P}_{\mathrm{i}}$ were resolved by thin layer chromatography (TLC) and radioactivity was detected by exposure to a film. A representative autoradiogram was shown on the top panel. The level of MT polymers was monitored at the 15-minute time point using an ultracentrifugation-based sedimentation-based assay. Samples from the supernatant (S) 
and pellet (P) fractions were resolved by SDS-PAGE and the gel stained by Coomassie blue. A representative gel is shown on the bottom panel. MTs were used at $2 \mu \mathrm{M}, \mathrm{KIF} 2 \mathrm{~A}-\mathrm{NM}$ at $50 \mathrm{nM}$ and DARPin at $1 \mu \mathrm{M}$. (B-C) Quantification of data from experiments shown in (A). B, data from autoradiograms; C, data from Coomassie blue stained gels. (D-F) Depolymerization of ${ }^{35} \mathrm{~S}$ GTP $\gamma$ S-labeled MTs at room temperature (ctrl), or by treatment of cold temperature $\left(4^{\circ} \mathrm{C}\right)$ or $\mathrm{Ca}^{2+}(1 \mathrm{mM})$ for 30 minutes. Samples were processed, quantified and analyzed in the way as those shown in (A-C). A representative autoradiogram and a Coomassie Blue stained gel were shown in (D), and their quantifications were shown in (E) and (F), respectively. All data represent the average of at least 3 independent experimental sets. Error Bars, S.D. (ns (not significant): $\mathrm{p}>0.05 ;{ }^{*} \mathrm{p} \leq 0.05 ;{ }^{*} \mathrm{p} \leq 0.01 ; * * * \mathrm{p} \leq 0.001$, by Student's $t$-test)

Fig. 3 | Stoichiometric relationship between kinesin-13 ATPase activity and the induced $\beta$ tubulin-GTP turnover.

(A) Kinesin-13-induced ATP hydrolysis and tubulin-GTP turnover were monitored using radiolabeled $\alpha^{32} \mathrm{P}$-ATP and $\gamma^{32} \mathrm{P}-\mathrm{GTP}$ as tracers in an enzymatic assay as described in Fig. 2. A representative autoradiogram of a TLC plate (out of 3 independent experimental runs) is shown. Tubulin dimers were used at $4 \mu \mathrm{M}$, KIF2A-NM and MCAK-NM at $500 \mathrm{nM}$, ATP and GTP at $200 \mu \mathrm{M}$. Reactions were carried out at room temperature for 10 minutes. (B-C) Quantification data for the experiments shown in (A). The levels of ATP and GTP hydrolysis were quantified based on the percentage of $\alpha^{32} \mathrm{P}-\mathrm{ADP}$ (from $\alpha^{32} \mathrm{P}-\mathrm{ATP}$ ) and ${ }^{32} \mathrm{P}_{\mathrm{i}}$ (from $\gamma^{32} \mathrm{P}-\mathrm{GTP}$ ) of the total amount of $\alpha^{32} \mathrm{P}$-ATP and $\gamma^{32} \mathrm{P}-\mathrm{GTP}$ used in the corresponding reaction. Data represent averages of at least 3 independent experimental sets. Error Bars, S.D.

\section{Fig. 4 | Conformational change of tubulin dimers precedes GTP hydrolysis as they incorporate into MT polymers.}

(A) The amount of tubulin-GTP hydrolyzed induced by different concentrations of paclitaxel (from $0-2 \mu \mathrm{M}$, as indicated) was measured over time (at 5, 10, $20 \mathrm{~min}$ ) at $4^{\circ} \mathrm{C}$, using a GDP detection assay. Tubulin dimers were used at $2 \mu \mathrm{M}$ with $50 \mu \mathrm{M}$ GTP. (B) MTs were polymerized using tubulin-GDP ( $5 \mu \mathrm{M}$ tubulin dimers and $200 \mu \mathrm{M}$ GDP) in the absence or presence of $20 \mu \mathrm{M}$ paclitaxel. The polymerization reactions were carried out at $37^{\circ} \mathrm{C}$ for $30 \mathrm{~min}$. The levels of MT polymerization were determined using an ultra-centrifugation-based sedimentation assay. 
Samples from the supernatant $(\mathrm{S})$ and pellet $(\mathrm{P})$ fractions were resolved by SDS-PAGE and the gel stained by Coomassie blue. A representative gel is shown. (C) The levels of polymerized MTs in (B) were quantified using ImageJ gel analysis. (D) Negative stained TEM images of MTs generated in (B). Scale bar, $1 \mu \mathrm{m}$. (E) The ATPase rate of KIF2A-NM in the presence of the MTs polymerized in (B) was determined using a Malachite green-based phosphate detection assay. (F) MTs were polymerized using $10 \mu \mathrm{M}$ tubulin dimers in the presence of $10 \%$ DMSO or $20 \mu \mathrm{M}$ paclitaxel (with $200 \mu \mathrm{M}$ GTP and the presence of a radio-labeled $\gamma^{32} \mathrm{P}$-GTP tracer). GTP hydrolysis was monitored during MT polymerization by quantifying the level of $\gamma^{32} \mathrm{P}_{\mathrm{i}}$ generated, as described in Fig. 3. The unpolymerized tubulin dimers and polymerized MTs were separated into supernatant $(\mathrm{S})$ and pellet $(\mathrm{P})$ fractions through ultra-centrifugation. To detect the level of $\gamma^{32}$ P-GTP more readily, the pellet fractions were loaded at $5 \mathrm{x}$ equivalence of the amount of the corresponding supernatant fractions. A representative autoradiogram from three independent experimental runs is shown. (G-H) Quantifications of $\gamma^{32} \mathrm{P}_{i}$ in the supernatant fractions (indicative of overall GTP hydrolysis) and $\gamma^{32} \mathrm{P}-\mathrm{GTP}$ in the pellet fractions (indicative of the amount of GTP on MT polymers) from the samples in F were shown in $(\mathrm{G})$ and $(\mathrm{H})$, respectively. (I-J) The levels of MT polymerization in reactions shown in (F) at $20 \mathrm{~min}$ were determined using a sedimentation assay. Samples were resolved by SDS-PAGE, stained and quantified in the same way as described above in (B-C). A representative Coomassie-blue stained gel (I) and the corresponding quantification ( $\mathrm{J}$ ) from three independent experiments were shown. Error bars, S.D. (ns (not significant): $\mathrm{p}>0.05 ;{ }^{*} \mathrm{p} \leq 0.05 ;{ }^{*} \mathrm{p} \leq 0.01 ;{ }^{* *} \mathrm{p} \leq 0.001$, by Student's $t$-test)

\section{Fig. 5 | The level of GTP-bound tubulin dimers incorporation into MT polymers is directly proportional to the number of polymerizing MT ends.}

(A) An illustration depicting MTs polymerized from different GMP-CPP seeds. Briefly, MT polymerization reactions were set up with $5 \mu \mathrm{M}$ tubulin and $200 \mu \mathrm{M}$ GTP alone (ctrl) or in the presence of short (R1) and long (R2) GMP-CPP seeds (at $1 \mu \mathrm{M}$ ) or shorter seeds generated by shearing of long seeds through a 25-gauge needle 5 time (R2' 5x) or 10 times (R2' 5x). (B-F) GTP hydrolysis level was assessed using radio-labeled $\gamma^{32} \mathrm{P}-\mathrm{GTP}$ as a tracer during the polymerization of MTs under the indicated reaction conditions as described in (A). Samples were processed the same way as described in Fig. 4F-J. (B) A representative autoradiogram is shown. (C-D) The corresponding quantifications of overall GTP hydrolysis, as indicated by the amount 
of $\gamma^{32} \mathrm{P}_{\mathrm{i}}$ in the supernatant fractions $(\mathrm{C})$, and of level of tubulin-GTP incorporation into MT polymers, as marked by $\gamma^{32} \mathrm{P}-\mathrm{GTP}$ in the pellet fractions (D). (E) The levels of MT polymerization in the reactions shown in (B) were measured using a sedimentation-based assay. A representative Coomassie-blue stained gel is shown. (F) The corresponding quantification of MT polymerization reactions shown in (E). (G) Negative stained TEM images of MTs generated from the indicated reactions as depicted in (A) and as carried out in (B). Scale bar, $1 \mu \mathrm{m}$. (H-H') Length distributions of MTs polymerized under the indicated reaction conditions, represented by scattered plots $(\mathrm{H})$ or binned histograms $\left(\mathrm{H}^{\prime}\right)$. The lines in $\mathrm{H}$ indicates the mean length, while the peaks in $\mathrm{H}^{\prime}$ marks the median length. Data represent averages of at least 3 independent experimental sets. Error Bars, S.D. (ns (not significant): $\mathrm{p}>0.05 ;{ }^{*} \mathrm{p} \leq 0.05 ;{ }^{*} \mathrm{p} \leq 0.01 ; * * * \mathrm{p} \leq 0.001$, by Student's $t$-test)

Fig. 6 | A model of conformational change-driven $\beta$-tubulin-GTP hydrolysis.

An illustration depicts MTs and tubulin dimers undergoing depolymerization and polymerization. This illustration also depicts the conformational changes of tubulin dimers from their native curved (GTP or GDP-bound) to a straighter state (within MT polymer) during polymerization or to an extreme curved structure (bound to kinesin-13) during depolymerization and back to their native curvature (upon released from kinesin-13). The curvature of $\alpha \beta$-tubulin dimers is marked by a yellow line going through the representative tubulin dimers. Note that GTP hydrolysis only occurs when $\alpha \beta$-tubulin dimers undergo conformational changes from a more curved conformation to a straighter one (from right to left), but not vice versa.

\section{Table 1 | Stoichiometric and Interdependent relationship between Kinesin-13 ATPase rates and $\beta$-tubulin-GTP turnover rates.}

The rates of Kinesin-13 ATP hydrolysis and tubulin-GTP turnovers were determined using two independent methods: radio-labeled $\alpha^{32} \mathrm{P}-\mathrm{ATP}, \gamma^{32} \mathrm{P}-\mathrm{GTP}$ and ${ }^{35} \mathrm{~S}-\mathrm{GTP} \gamma \mathrm{S}$ in an enzymatic assay (as shown in Fig. 3 and Fig. S6); and Malachite green-based phosphate detection assay (shown in Fig. S7). The levels of ATP hydrolysis of kinesin-13s (KIF2A-NM and MCAK-NM) were quantified for different nucleotide-bound tubulins. (* To calculate the rate of GTP hydrolysis, we had to use ATP $\gamma \mathrm{S}$ instead of ATP.) 
Figure 1 (which was not certified by peer review) is the author/funder. All rights reserved. No reuse allowed without permission.

A

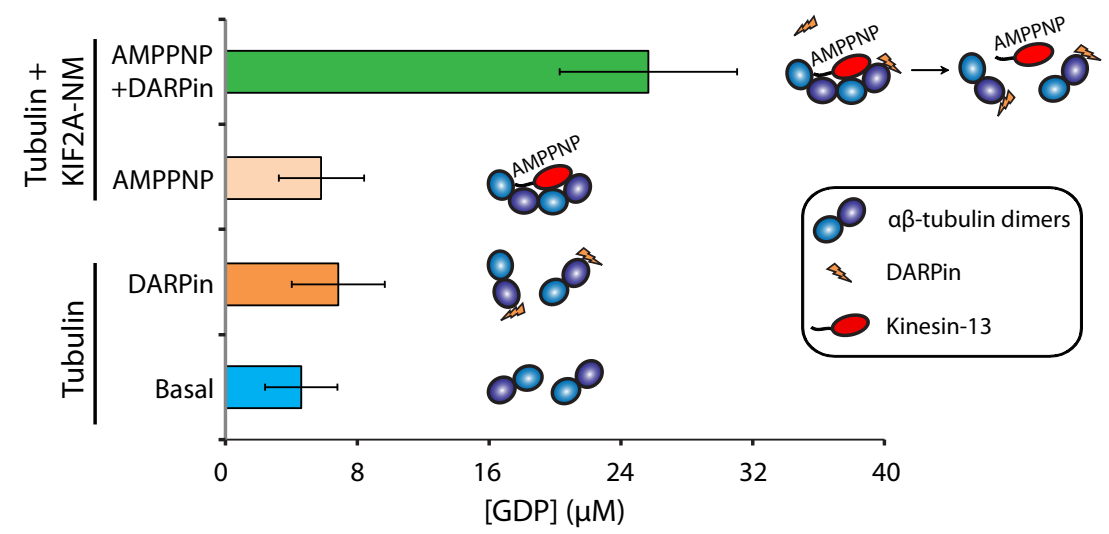

B

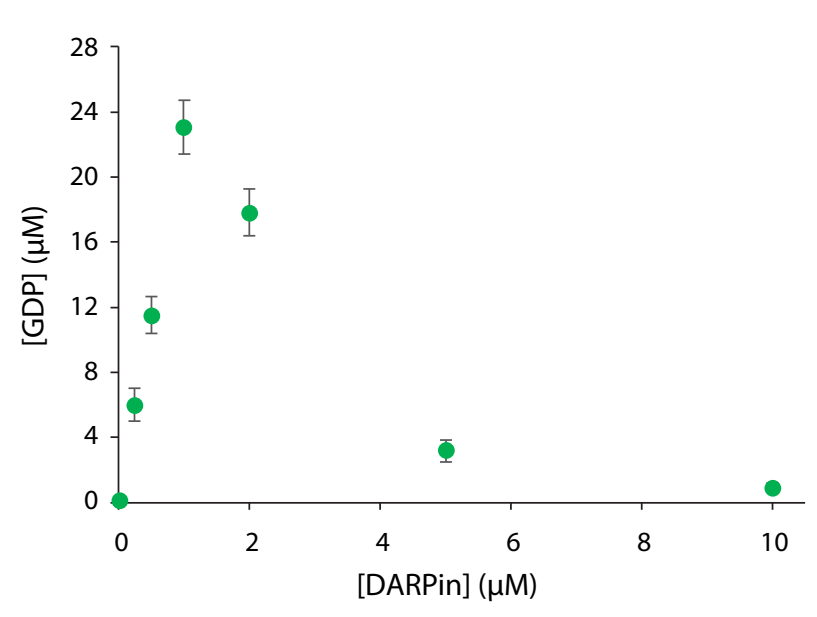

D

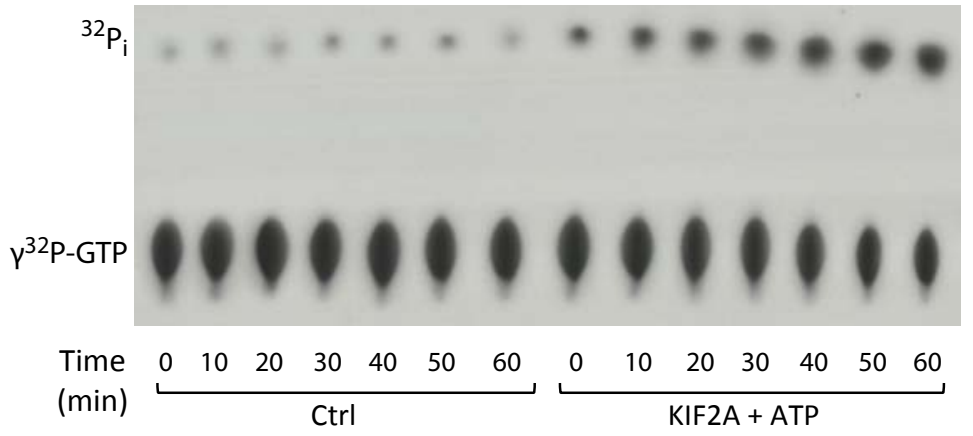

C

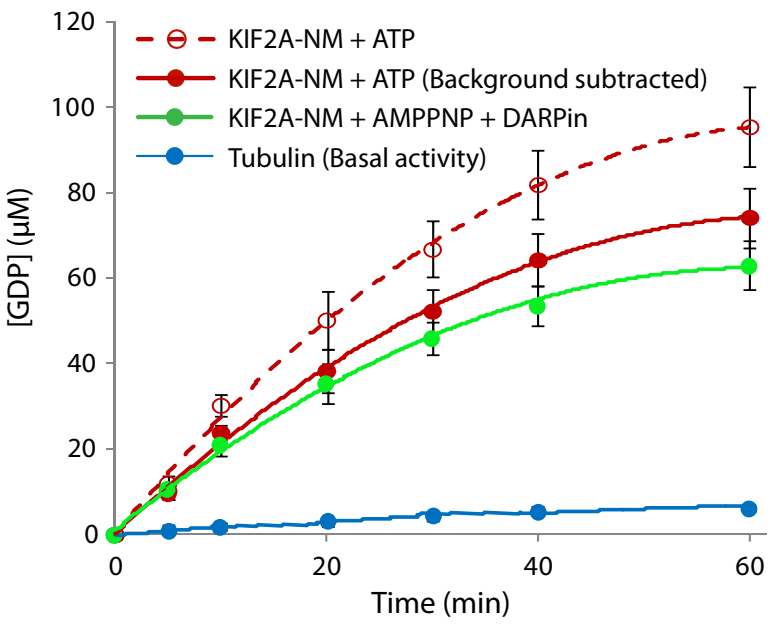

E

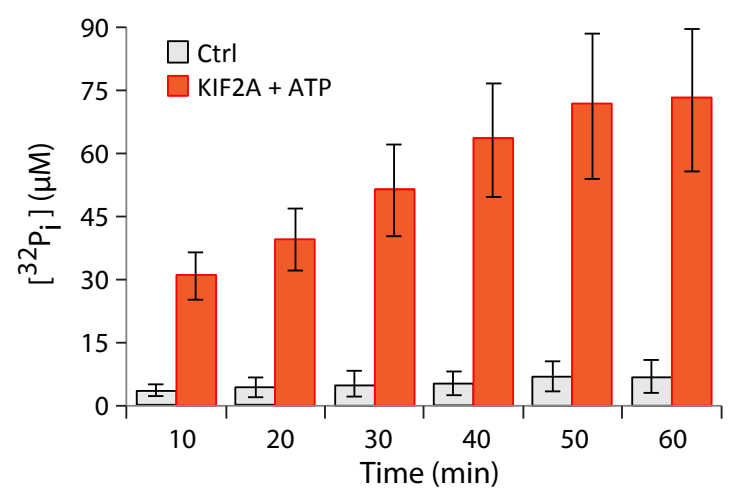


A

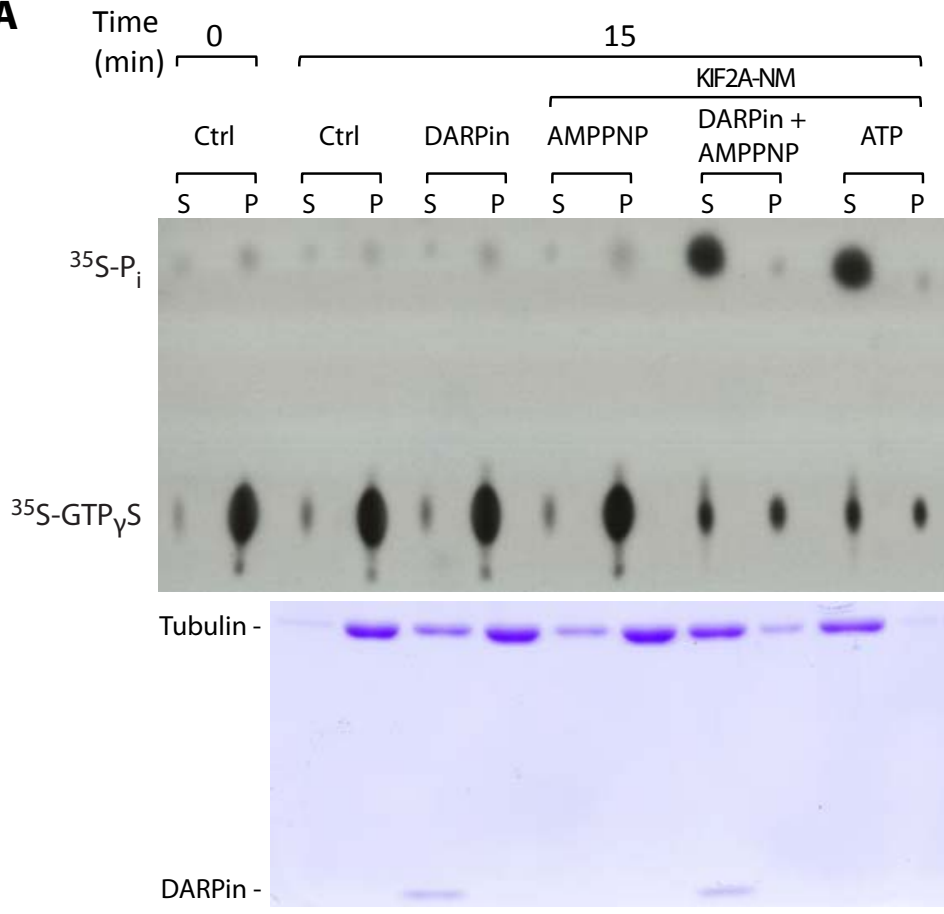

D

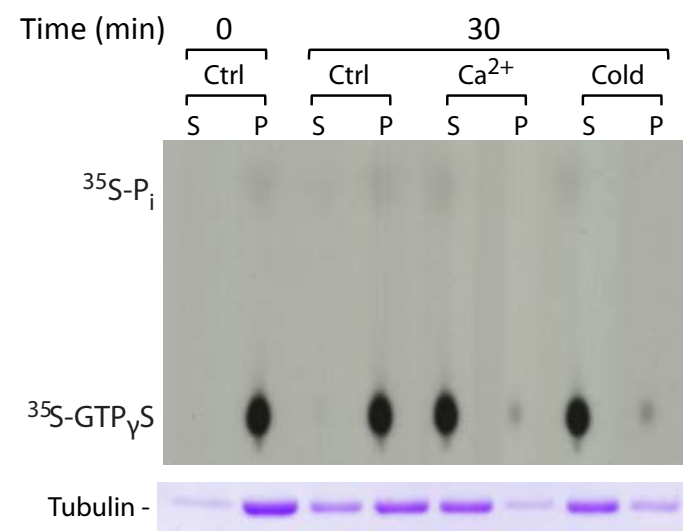

B

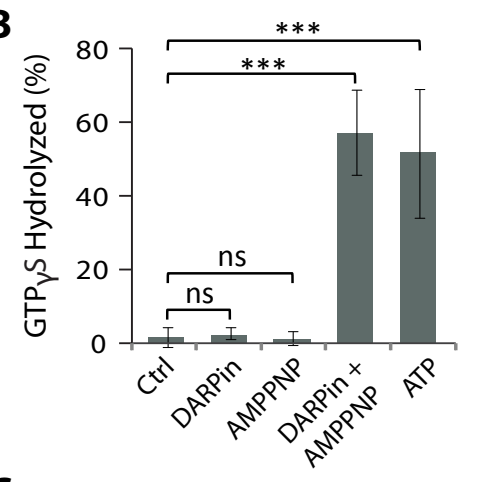

C

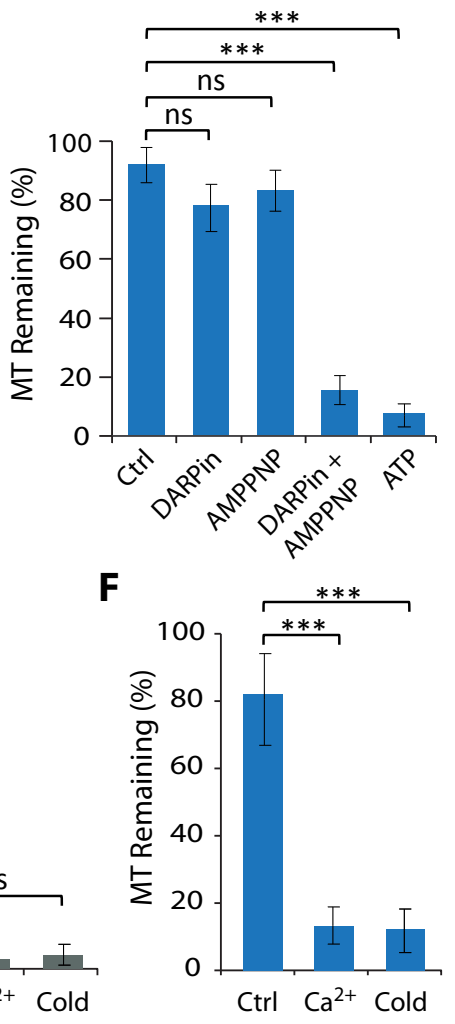


bioRxiv preprint doi: https://doi.org/10.1101/2020.09.08.288019; this version posted September 10, 2020. The copyright holder for this preprint

Fig Ure 3 (which was not certified by peer review) is the author/funder. All rights reserved. No reuse allowed without permission.

A

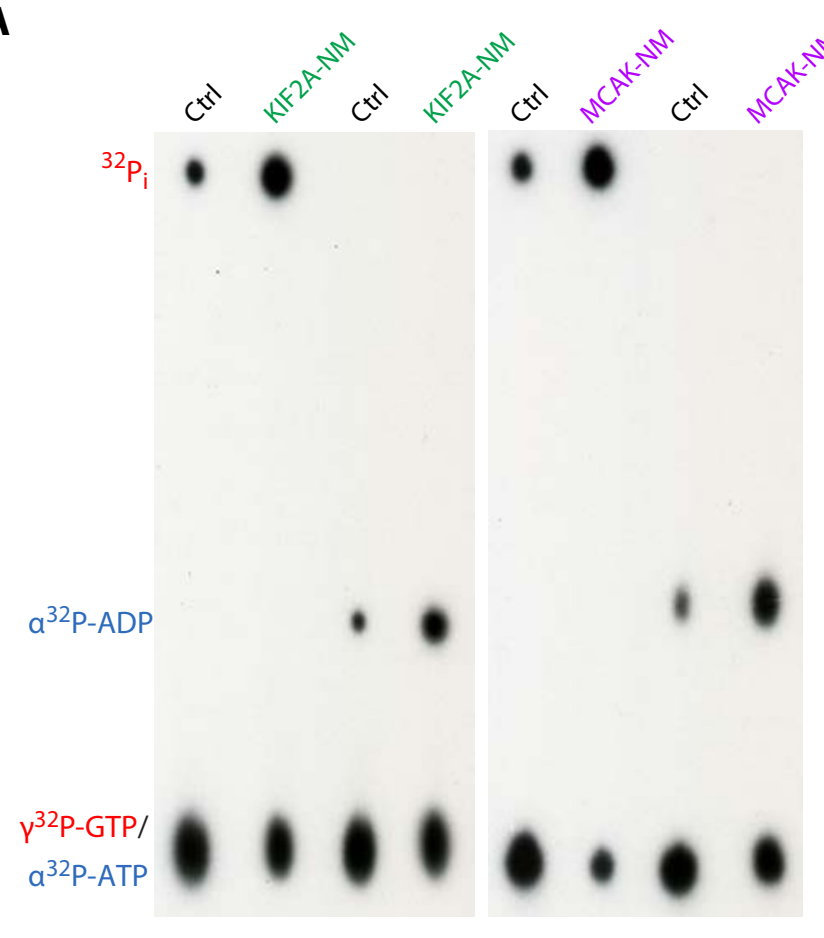

B

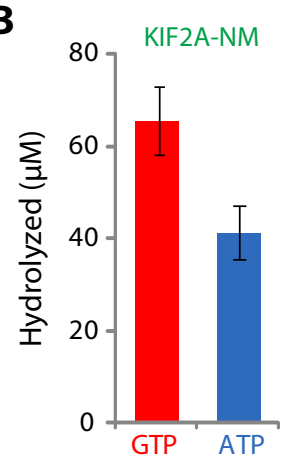

C

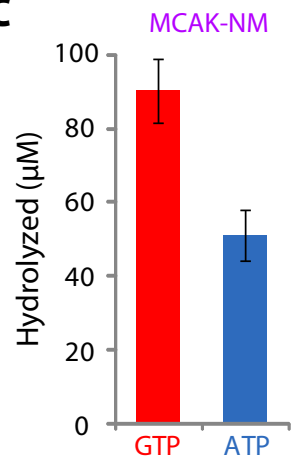


A

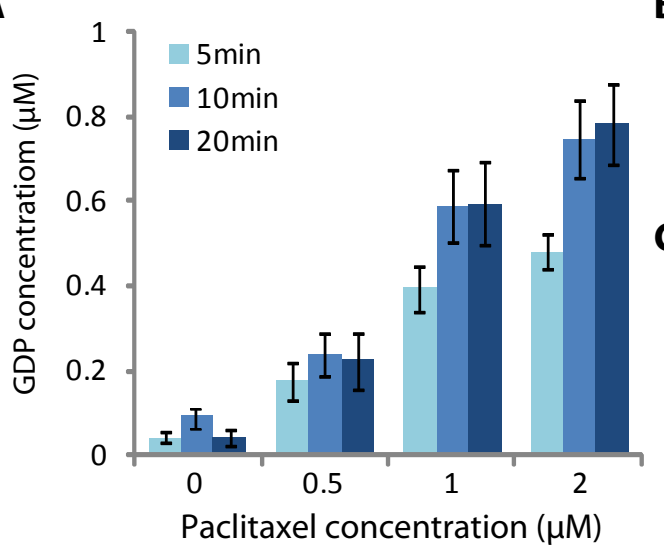

$\mathbf{F}$

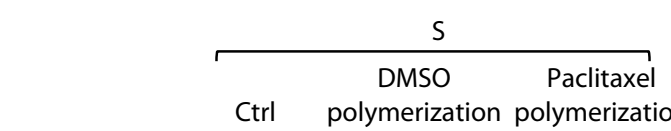

B

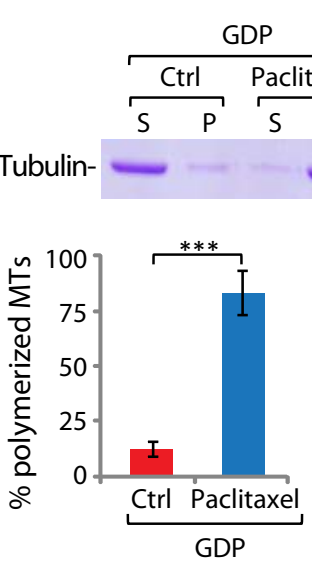

D

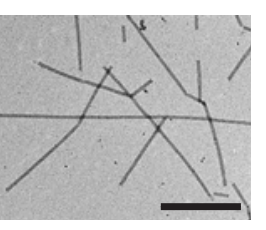

E

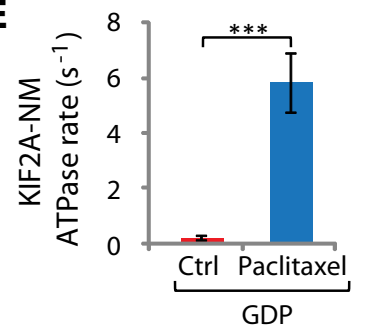

Ctrl polymerization polymerization

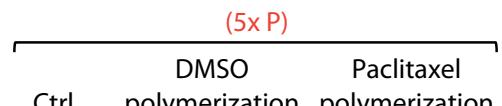

Time (min)

${ }^{32} \mathrm{P}_{\mathrm{i}}$

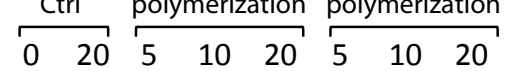

$\mathrm{Y}^{32 \mathrm{P}-\mathrm{GTP}}$

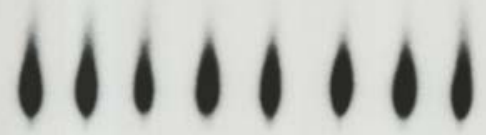

G

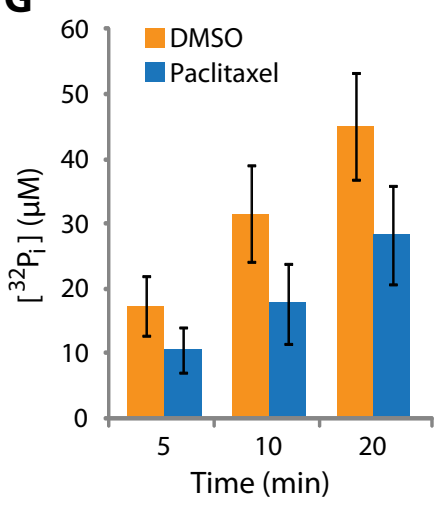

H

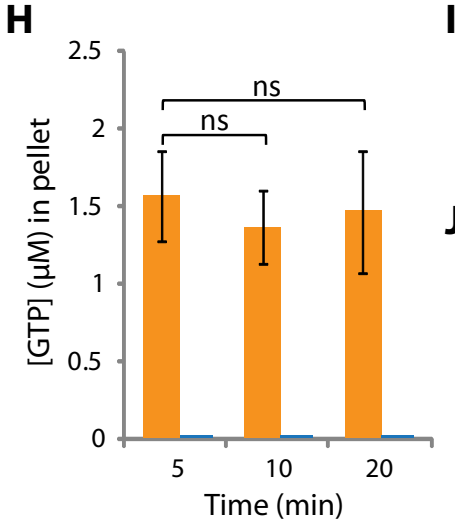

I

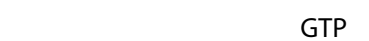

Tubulin -

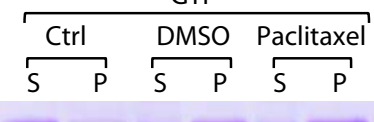

J

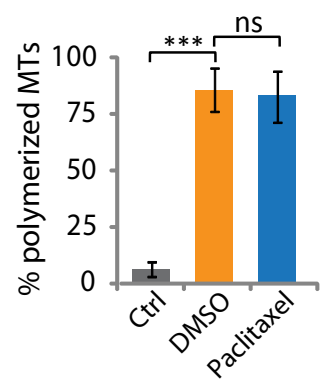


bioRxiv preprint doi: https://doi.org/10.1101/2020.09.08.288019; this version posted September 10, 2020. The copyright holder for this preprint

Figure 5 (which was not certified by peer review) is the author/funder. All rights reserved. No reuse allowed without permission.

A

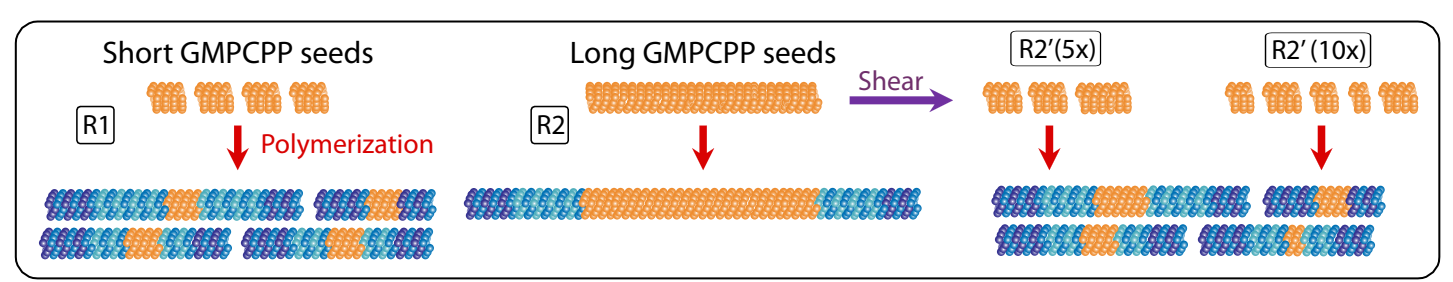

B

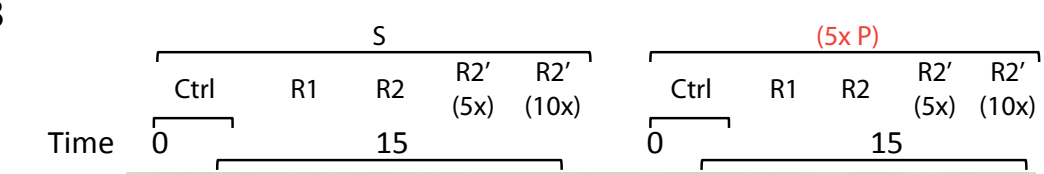

Tubulin -

F

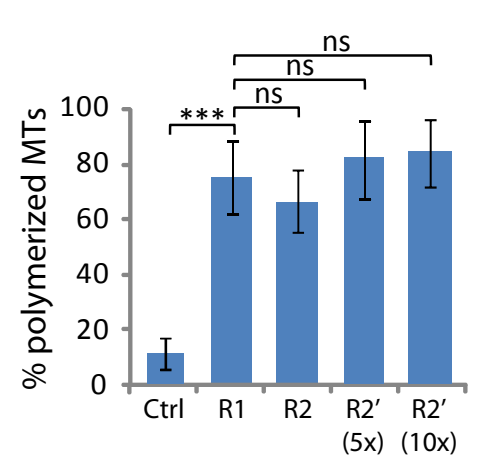

H

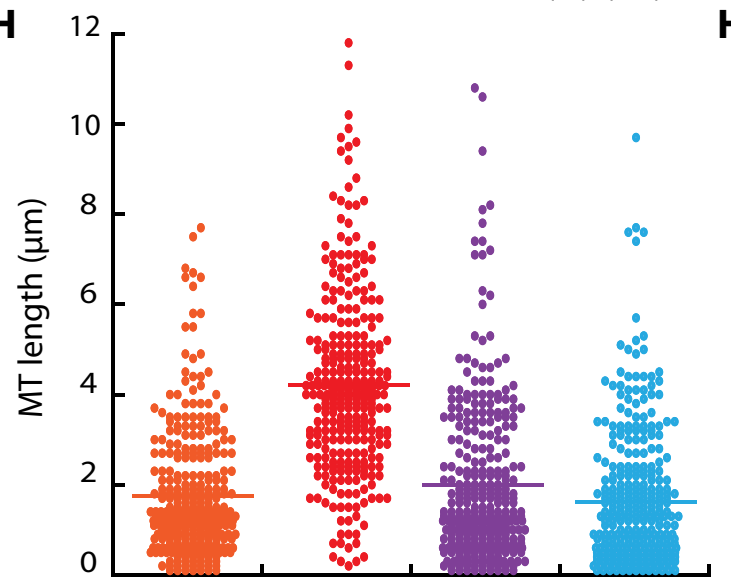

E

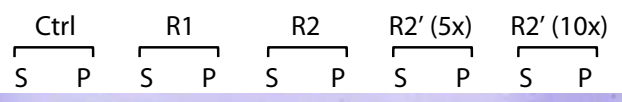

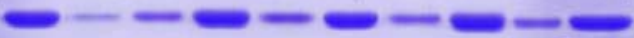

$H^{\prime}$
C
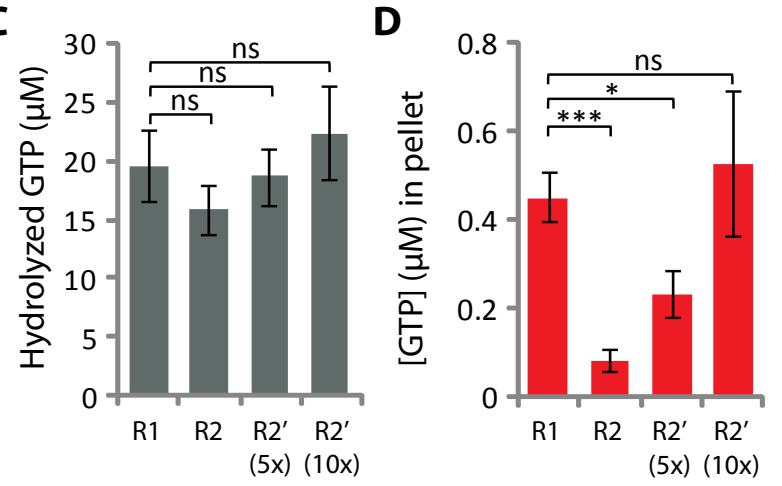

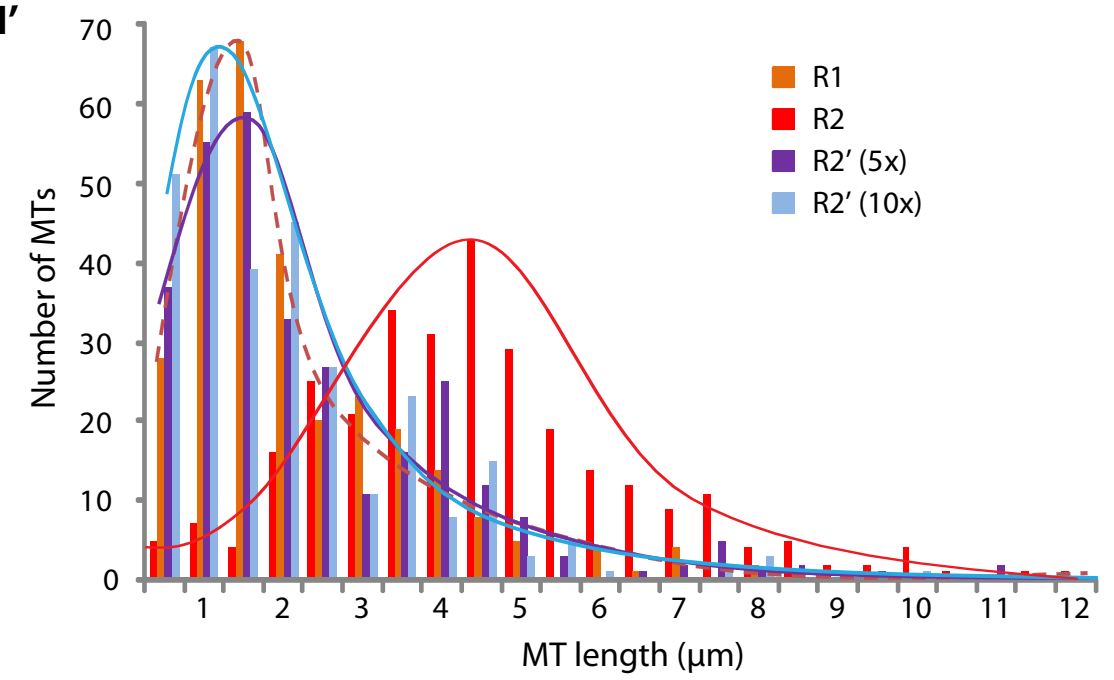


CD bioRxiv preprint doi: https://doi.org/10.1101/2020.09.08.288019; this version posted September 10, 2020. The copyright holder for this preprint Figure 6 (which was not certified by peer review) is the author/funder. All rights reserved. No reuse allowed without permission.

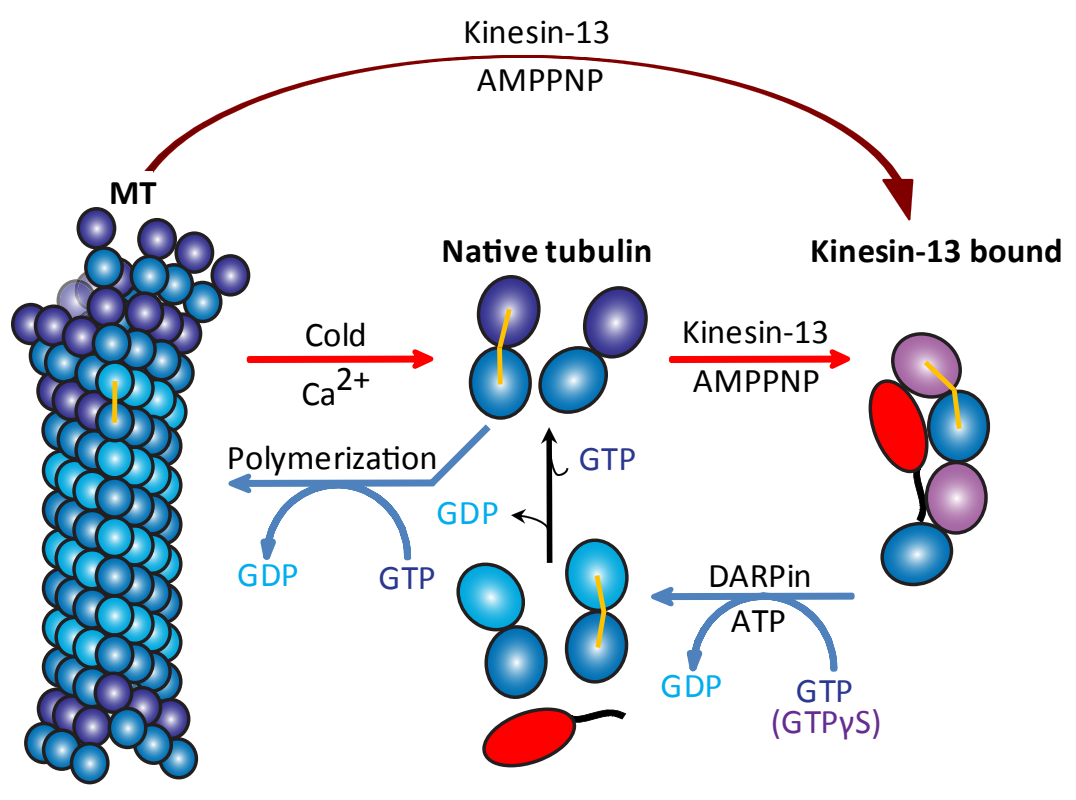


bioRxiv preprint doi: https://doi.org/10.1101/2020.09.08.288019; this version posted September 10, 2020. The copyright holder for this preprint

Table 1

\begin{tabular}{|c|c|c|c|c|c|}
\hline & \multirow[t]{3}{*}{ Nucleotide state } & \multicolumn{4}{|c|}{ Hydrolysis rate $\left(\mathrm{s}^{-1}\right)$} \\
\hline & & \multicolumn{2}{|c|}{ Hot assay } & \multicolumn{2}{|c|}{ Malachite green } \\
\hline & & ATP & GTP & ATP & GTP \\
\hline \multirow{4}{*}{ 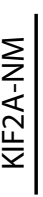 } & Tub-GTP & $0.13 \pm 0.04$ & $0.23 \pm 0.06$ & $0.16 \pm 0.04$ & $0.31 \pm 0.09 *$ \\
\hline & Tub-GTPץS & $0.14 \pm 0.04$ & $0.26 \pm 0.07$ & $0.18 \pm 0.05$ & - \\
\hline & Tub-GDP & $0.09 \pm 0.03$ & - & $0.12 \pm 0.04$ & - \\
\hline & Tub-APO & $0.11 \pm 0.02$ & - & $0.11 \pm 0.03$ & - \\
\hline \multirow{4}{*}{ 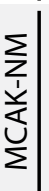 } & Tub-GTP & $0.15 \pm 0.04$ & $0.29 \pm 0.06$ & $0.17 \pm 0.05$ & $0.33 \pm 0.07$ \\
\hline & Tub-GTPץS & $0.16 \pm 0.04$ & $0.31 \pm 0.06$ & $0.18 \pm 0.05$ & - \\
\hline & Tub-GDP & $0.11 \pm 0.04$ & - & $0.13 \pm 0.03$ & - \\
\hline & Tub-APO & $0.10 \pm 0.03$ & - & $0.13 \pm 0.04$ & - \\
\hline
\end{tabular}

